Mechanism of Action of

\title{
Small-Molecule Agents in Ongoing Clinical Trials for SARS-CoV-2: A Review
}

\author{
Lei Zhao ${ }^{1,2}$, Song $L^{1 *}$ and Wu Zhong ${ }^{1 *}$ \\ ${ }^{1}$ National Engineering Research Center for the Emergency Drug, Beijing Institute of Pharmacology and Toxicology, Beijing, China, \\ ${ }^{2}$ Beijing Sunho Pharmaceutical Co., Ltd., Beijing, China
}

Since the first reports from December 2019, COVID-19 caused an overwhelming global pandemic that has affected 223 countries, seriously endangering public health and creating an urgent need for effective drugs to treat SARS-CoV-2 infection. Currently, there is a lack of safe, effective, and specific therapeutic drugs for COVID-19, with mainly supportive and symptomatic treatments being administered to patients. The preferred

OPEN ACCESS

Edited by:

Veria Khosrawipour,

Cornell University, United States

Reviewed by: Agata Mikołajczyk-Martinez, Wroclaw University of Environmental and Life Sciences, Poland

Partha Karmakar,

Abzena, United Kingdom

*Correspondence: Song Li

lis.lisong@gmail.com

Wu Zhong

zhongwu@bmi.ac.cn

Specialty section:

This article was submitted to

Experimental Pharmacology and Drug

Discovery,

a section of the journal Frontiers in Pharmacology

Received: 21 December 2021 Accepted: 28 January 2022

Published: 25 February 2022

Citation:

Zhao L, Li S and Zhong W (2022) Mechanism of Action of SmallMolecule Agents in Ongoing Clinical Trials for SARS-CoV-2: A Review.

Front. Pharmacol. 13:840639. doi: 10.3389/fphar.2022.840639 option for responding to an outbreak of acute infectious disease is through drug repurposing, saving valuable time that would otherwise be lost in preclinical and clinical research, hastening clinical introduction, and lowering treatment costs. Alternatively, researchers seek to design and discover novel small-molecule candidate drugs targeting the key proteins in the life cycle of SARS-CoV-2 through an in-depth study of the infection mechanism, thus obtaining a number of candidate compounds with favorable antiviral effects in preclinical and clinical settings. There is an urgent need to further elucidate the efficacy and mechanism of action of potential anti-SARS-CoV-2 smallmolecule drugs. Herein, we review the candidate small-molecule anti-SARS-CoV-2 drugs in ongoing clinical trials, with a major focus on their mechanisms of action in an attempt to provide useful insight for further research and development of small-molecule compounds against SARS-CoV-2 infection.

Keywords: mechanism of action, SARS-CoV-2, small-molecule drug, antiviral, COVID-19, clinical

\section{INTRODUCTION}

Since its identification in December 2019, severe acute respiratory syndrome coronavirus 2 (SARSCoV-2) has spread, resulting in a global pandemic of coronavirus disease 2019 (COVID-19), an acute respiratory infection posing a severe threat to human health and public health security on a global scale (Wang C. et al., 2020; Zhou et al., 2020). With the continuous spread of SARS-CoV-2, various new variants have emerged, the four most notable ones being alpha, beta, gamma, and delta, which have caused recent outbreaks in many countries and regions, thus representing a major challenge in the fight to curb the pandemic (Davies et al., 2021; Faria et al., 2021; World Health Organization, 2021). According to the most recent statistics from Johns Hopkins University, as of 4 November 2021, there are 248,625,178 confirmed cases of COVID-19 infection, which has claimed 5,029,325 lives across 223 countries worldwide (Coronavirus Resource Center, 2021). 
In the face of the COVID-19 pandemic, scientists are actively engaged in researching and developing effective drugs against SARS-CoV-2. The preferred strategy in conditions of an acute infectious disease outbreak is to rapidly repurpose existing effective drugs, thus saving time for preclinical/clinical research and significantly reducing the time and cost associated with drug development (Wang M. et al., 2020; Talevi and Bellera, 2020). Alternatively, researchers have sought to design and discover novel small-molecule drugs targeting key proteins in the SARS-CoV-2 life cycle through an in-depth study of the mechanisms underlying infection, with a number of small-molecule candidate drugs already described (Dai et al., 2020; Yin et al., 2020). At present, only remdesivir and molnupiravir have been approved by the FDA and MHRA, respectively, for the treatment of COVID-19. Several candidate drugs have also shown promising antiviral efficacy in preclinical and clinical trials. Nevertheless, there is an urgent need to comprehensively elucidate the mechanism of action of these small-molecule drugs, identify potential drug targets, and develop more effective and safer anti-SARS-CoV-2 agents.

\section{SARS-COV-2: BIOLOGICAL CHARACTERISTICS, LIFE CYCLE, AND DRUG TARGETS}

SARS-CoV-2 is a single-stranded, positive-sense, enveloped RNA virus, approximately $60-140 \mathrm{~nm}$ in diameter (Zhu N. et al., 2020). It belongs to the genus $\beta$-coronavirus in the Coronaviridae family, sharing $79 \%$ sequence homology with SARS-CoV, yet having a higher transmission capacity (Lu et al., 2020; Xu et al., 2020). The genome consists of approximately 29,900 bases and encodes 29 proteins, including 16 non-structural proteins (NSPs), with at least 13 downstream open-reading frames (ORFs) (Wu F. et al., 2020). Among NSPs, papain-like protease and 3C-like protease (3CLpro, NSP5) cleave ppla and pp1b polypeptides into at least 16 NSPs. NSP7, NSP8, and NSP12 constitute RNA-dependent RNA polymerases responsible for SARS-CoV-2 replication and transcription. The downstream coding region mainly encodes structural proteins, including spike (S), membrane (M), envelope (E), nucleocapsid $(\mathrm{N})$ protein, and several accessory proteins (Zhu G. et al., 2020).

The SARS-CoV-2 infection cycle is divided into several steps: attachment, entry (endocytosis and membrane fusion), RNA and protein synthesis, virion assembly, and release. As with most viruses, attachment to host cells is the first step initiating infection. The $S$ protein receptor-binding domain interacts with host cell surface receptor proteins to facilitate attachment. The main host cell receptors involved are the angiotensinconverting enzyme 2 (ACE2), widely expressed in various tissues and organs, and the recently reported CD147-spike protein (Hoffmann et al., 2020a; Wang K. et al., 2020). After attachment, SARS-CoV-2 entry is achieved via endocytosis and/ or direct membranes fusion, with S2 being cleaved by different proteases depending on the entry pathway. In the presence of TMPRSS2, the S protein undergoes proteolytic cleavage by host proteases TMPRSS2 and TMPRSS11D, allowing for viral-host membrane fusion. The SARS-CoV-2-ACE2 complex is internalized into endosomes via clathrin-mediated endocytosis in the absence of TMPRSS2, where S2 is cleaved by cathepsin L in the acidic environment to facilitate membrane fusion (Jackson et al., 2021). In both entry patterns, enzymatic cleavage discloses the fusion peptide and induces conformational changes in the S2 subunit, driving the peptide to move into the membrane and thus launching membrane fusion (Jackson et al., 2021). Genomic nucleic acid is then released into the cytoplasm for the following replication and translation. The genomic RNA acts as a template and attaches to ribosomes to translate viral proteases and polymeric protein precursors, which are then cleaved by 3CLpro and PLpro, respectively, to form functional NSPs. NSP12, NSP7, and NSP8 form the SARS-CoV-2 RNAdependent RNA polymerase (RdRp) complex, responsible for the replication and transcription of genomic RNA in the cytoplasm (Gao et al., 2020). At the same time, the subgenomic mRNA is translated into structural and accessory proteins, utilizing the host translation machinery. In the assembly and release stage, the nucleocapsid, formed through the assembly of genomic RNA and the $\mathrm{N}$ protein, assembles with envelope proteins to then form progeny virions (Chen et al., 2020). Finally, the virions are released from infected cells to continue the viral replication cycle.

Therefore, drugs targeting viral proteins related to the virus life cycle (Figure 1), including but not limited to 3CLpro, PLpro, and RdRp, as well as host-related target proteins, such as ACE2 and TMPRSS2, to block virus entry and replication, are expected to have therapeutic potential.

\section{SMALL-MOLECULE AGENTS IN ONGOING CLINICAL TRIALS FOR COVID-19}

Although the SARS-CoV-2 pandemic has slowed to some extent due to widespread vaccination, there is an urgent need for effective antiviral drugs. The results of the Solidarity Clinical Trial, a large-scale, randomized clinical study carried out by WHO, indicated that the four candidate drugs identified early in the outbreak (such as remdesivir) are ineffective or have little effect on COVID-19 (Pan et al., 2021). Antibody formulations and other therapies have not made satisfactory clinical progress either. Small-molecule oral agents have obvious advantages with regard to delivery and availability, with potential antiviral activity against mutant strains, highlighting their potential role in the fight against SARS-CoV-2. In addition to the FDA- and MHRAapproved remdesivir and molnupiravir, a total of 11 drugs and biologicals are authorized for emergency use, of which only baricitinib is a small-molecule drug (Food and Drug Administration, 2021). There are 20 approved drugs for COVID-19 worldwide, and more than 380 candidate drugs are undergoing clinical development. In the present work, we review small-molecule agents at the clinical stage or approved drugs (as shown in Table 1) as well as their respective mechanisms of action against SARS-CoV-2. The discussed agents include, but are not limited to, the highly touted molnupiravir, AT-527, PF07321332, and S-217622. 


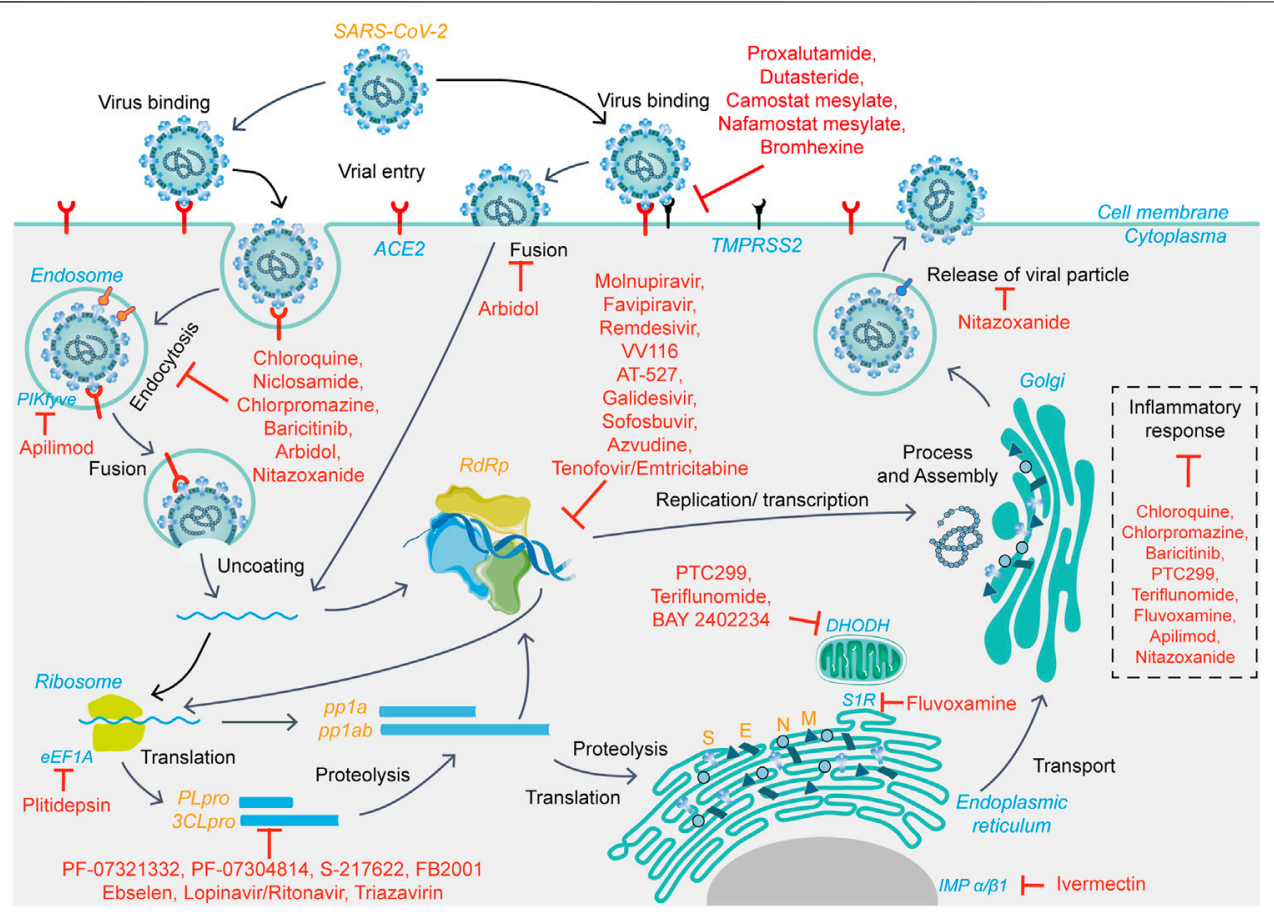

FIGURE 1 | The viral life cycle of SARS-CoV-2 and potential mechanisms of action of small-molecule candidate drugs.

\section{MECHANISM OF ACTION OF SMALL-MOLECULE AGENTS}

\subsection{Antiviral Agents Targeting Viral Proteins 4.1.1 Inhibitors of SARS-CoV-2 RdRp}

Remdesivir is a nucleoside analog developed by Gilead, with inhibitory effects against Ebola, MERS, and SARS viruses. The $\mathrm{EC}_{50}$ of remdesivir against SARS-CoV-2 was reported to be $0.77 \mu \mathrm{M}$ in Vero E6 cells (Wang X. et al., 2020). As a prodrug, remdesivir is converted to a triphosphate derivative by host enzymes. It then competitively binds the RdRp of SARS-CoV2, leading to its incorporation into the growing viral RNA chain and the premature termination of chain synthesis, that is, the inhibition of viral replication (Yin et al., 2020). Remdesivir is the first FDA-approved drug for the treatment of COVID-19, but it has become controversial with increasing clinical evidence questioning its efficacy. The poor in vivo efficacy of remdesivir may be attributed to the design based on hepatic targeting and the rate of conversion to the active form. Remdesivir is a phospholipid amide prodrug with the parent nucleoside GS-441524, which is designed for hepatic targeting and can be rapidly metabolized and converted in such cells. In contrast, the target cells of SARS-CoV-2 are mainly respiratory and lung-associated cells, which may not sufficiently metabolize the drug. In addition, remdesivir is converted to GS-441524 in plasma, and its initial conversion to monophosphate form is slow and considered as the rate-limiting step. VV116 is a novel nucleoside analog that is modified with remdesivir as a prototype, primarily through a prodrug strategy to improve its oral bioavailability and anti-
SARS-CoV-2 activity. The anti-SARS-CoV-2 EC $_{50}$ of VV116 was $0.35 \mu \mathrm{M}$ compared to $1.71 \mu \mathrm{M}$ for remdesivir in Vero E6 cells, and VV116 was also effective in reducing lung viral load and titer in a dose-dependent manner in a mouse infection model (Xie et al., 2021). Based on the preclinical results, the drug is in phase I clinical trials for the treatment of COVID-19 in Uzbekistan.

Favipiravir is a nucleoside analog developed by Toyama Chemical Co. Ltd., used to treat influenza in Japan and China. Favipiravir is a broad-spectrum antiviral drug, including influenza, arboviruses, filoviruses, bunyavirus, and coronaviruses. Favipiravir was reported to have an $\mathrm{EC}_{50}$ of $61.88 \mu \mathrm{M}$ against SARS-CoV-2 in Vero E6 cells (Wang M. et al., 2020). Several clinical trials have preliminarily demonstrated its efficacy and safety (Cai et al., 2020; Fu et al., 2020; Ivashchenko et al., 2021), with large-scale clinical trials currently underway (Appili Therapeutics Inc., 2020; PRINCIPLE, 2021). Favipiravir has been authorized for emergency COVID-19 treatment in several countries, such as Turkey, India, and Russia. Favipiravir is a prodrug and its active form, favipiravir ribofuranosyl-triphosphate, inhibits viral replication by targeting $\mathrm{RdRp}$. There are two main hypotheses regarding the action of favipiravir at present (Zhao and Zhong, 2021). Naydenova et al. reported that favipiravir ribofuranosyl-triphosphate, as a nucleoside analog, simulates guanosine triphosphate (GTP) or adenosine triphosphate (ATP) to be mixed into the RNA of nascent viruses by the SARS-CoV-2 RdRp complex, inducing the termination of RNA synthesis (Naydenova et al., 2021). In contrast, several studies based on the structural analysis of 
TABLE 1 | General information of anti-SARS-CoV-2 drugs in clinical development.

\begin{tabular}{|c|c|c|c|c|c|}
\hline Drugs & $\begin{array}{l}\text { No. of } \\
\text { clinical trials } \\
\text { registered }^{\mathrm{a}}\end{array}$ & Phase & Molecular target & Development strategy & $\begin{array}{l}\text { Approval status } \\
\text { (for COVID-19) }\end{array}$ \\
\hline Remdesivir & 77 & 4 & $\mathrm{RdRp}$ & Repurposing & Approval by FDA \\
\hline Favipiravir & 46 & 4 & $\mathrm{RdRp}$ & Repurposing & $\begin{array}{l}\text { EUA in several } \\
\text { countries }\end{array}$ \\
\hline Molnupiravir & 5 & 3 & $R d R p$ & Novel & $\begin{array}{l}\text { Approval by } \\
\text { MHRA; EUA by FDA }\end{array}$ \\
\hline AT-527 & 3 & 3 & $\mathrm{RdRp}$ & Novel & Non-approved \\
\hline Galidesivir & 1 & 1 & $\operatorname{RdRp}$ & Repurposing & Non-approved \\
\hline Sofosbuvir & 8 & 4 & $\mathrm{RdRp}$ & Repurposing & Non-approved \\
\hline Azvudine & 3 & 3 & $\mathrm{RdRp}$ & Repurposing & Non-approved \\
\hline Tenofovir/emtricitabine & 5 & 3 & $\mathrm{RdRp}$ & Repurposing & Non-approved \\
\hline PF-07321332 & 8 & 3 & 3CLpro & Novel & EUA by FDA \\
\hline PF-07304814 & 3 & 1 & 3CLpro & Novel & Non-approved \\
\hline s-217622 & - & $2 / 3$ & 3CLpro & Novel & Non-approved \\
\hline FB2001 & 1 & $2 / 3$ & 3CLpro & Novel & Non-approved \\
\hline Ebselen & 2 & 2 & 3CLpro & Repurposing & Non-approved \\
\hline Lopinavir/ritonavir & 24 & 4 & 3CLpro & Repurposing & Non-approved \\
\hline Triazavirin & 2 & 4 & RNA synthesis/3CLpro & Repurposing & Non-approved \\
\hline Chloroquine/hydroxychloroquine & $46 / 276$ & 4 & Endosomal entry & Repurposing & $\begin{array}{l}\text { EUA by FDA at } \\
\text { earlier outbreak } \\
\text { (chloroquine) }\end{array}$ \\
\hline Umifenovir/arbidol & 3 & 4 & Endosomal entry & Repurposing & Non-approved \\
\hline Niclosamide & 11 & 3 & Endosomal entry & Repurposing & Non-approved \\
\hline Chlorpromazine & 2 & 3 & Endosomal entry & Repurposing & Non-approved \\
\hline Baricitinib & 20 & 4 & Endosomal entry & Repurposing & EUA by FDA \\
\hline Proxalutamide & 5 & 3 & Androgen receptor antagonist & Repurposing & Non-approved \\
\hline Dutasteride & 1 & 2 & 5-alpha-reductase inhibitor & Repurposing & Non-approved \\
\hline Camostat mesylate & 5 & 3 & TMPRSS2 inhibitor & Repurposing & Non-approved \\
\hline Nafamostat mesylate & 2 & 2 & TMPRSS2 inhibitor & Repurposing & Non-approved \\
\hline PTC299 & 1 & 2 & $\mathrm{DHODH}$ inhibitor & Repurposing & Non-approved \\
\hline Teriflunomide & 3 & 3 & DHODH inhibitor & Repurposing & Non-approved \\
\hline Nitazoxanide & 23 & 4 & Endosomal entry/Inflammatory response regulation & Repurposing & Non-approved \\
\hline Fluvoxamine & 1 & 3 & Sigma-1 receptors agonist & Repurposing & Non-approved \\
\hline Plitidepsin & 3 & 3 & eEF1A inhibitor & Repurposing & Non-approved \\
\hline Ivermectin & 69 & 4 & IMPA $/ \beta 1$ inhibitor & Repurposing & Non-approved \\
\hline Apilimod & 1 & 2 & PIKFYV inhibitor & Repurposing & Non-approved \\
\hline
\end{tabular}

${ }^{a}$ Registered on ClinicalTrials.gov.

viral RdRp-drug complexes showed that favipiravir ribofuranosyl-triphosphate mimicked the incorporation of ATP and GTP nucleotides into nascent RNA products and induced high rates of genomic mutation that led to the production of non-viable virions, further inhibiting viral replication and reproduction (Shannon et al., 2020; Peng et al., 2021; Zhao and Zhong, 2021). In the presence of intracellular ATP or GTP, the termination of RNA synthesis is unlikely. Favipiravir and remdesivir have different antiviral mechanisms, which may be due to the differences in their influence on RdRp, although they both inhibit its activity.

Molnupiravir (EIDD-2801) is an isopropyl prodrug of the nucleoside analog $\beta$-D-N4-hydroxycytidine that targets the RdRp. It displays a broad-spectrum antiviral activity, including influenza, Ebola, and coronaviruses. The $\mathrm{IC}_{50}$ of molnupiravir against SARS-CoV-2 was 0.3 and $0.08 \mu \mathrm{M}$ in Vero and Calu-3 cells, respectively, with a $\mathrm{CC}_{50}>10 \mu \mathrm{M}$. Molnupiravir effectively prevents and treats SARS-CoV-2, SARS-CoV, and MERS-CoV infections in mice (Sheahan et al., 2020; Wahl et al., 2021). In ferret models, molnupiravir effectively reduced viral load and completely prevented virus transmission through direct contact with untreated animals (Cox et al., 2021). Several clinical trials have been conducted on molnupiravir as a potential agent for COVID-19 treatment. In April 2021, Merck announced discontinuing studies in hospitalized patients due to a lack of efficacy. In early October and November, Merck and Ridgeback Biotherapeutics announced interim data and additional analyses, respectively, from a clinical trial (NCT04575597, MOVe-OUT) of molnupiravir for the treatment of patients with mild or moderate COVID-19. In the analysis, $6.8 \%$ of molnupiravir-treated patients were either hospitalized or died (48/709) following randomization, compared with $9.7 \%$ of patients who received placebo (68/699) through day 29 ( $p=$ 0.0218). Molnupiravir reduced the risk of hospitalization or death by approximately 30\% (Merck, 2021a). The MHRA has granted authorization for molnupiravir in the United Kingdom, making it the first oral antiviral drug for treating adult patients with mild or moderate COVID-19 and at least one risk factor for developing severe illness (Merck, 2021b). 
According to systematic biochemical and structural analyses, Kabinger et al. (2021) suggested that molnupiravir exerts its antiviral effects by inducing SARS-CoV-2 RNA mutagenesis and proposed a two-step model. NHC triphosphate, the active form of molnupiravir, can be misidentified by the RdRp of SARSCoV-2 as CTP or UTP during RNA synthesis. Therefore, when the positive-strand genomic RNA (+gRNA) is used by SARSCoV-2 RdRp as a template to synthesize subgenomic RNA (sgRNA) and negative-strand gRNA, in the first step, $M$ is incorporated into sequences instead of $\mathrm{U}$ or $\mathrm{C}$. In the next step, the resulting M-containing negative-strand gRNA is used as a template for synthesizing +gRNA, which results in mutations in these newly synthesized RNAs. This mutagenesis of RNA is predicted not to maintain the formation of intact progeny viruses. This conclusion is consistent with previous results on the mechanism of molnupiravir against other viruses. Taken together, molnupiravir, as a mutagen, causes "error disasters" during viral replication, thereby exerting an antiviral effect (Sheahan et al., 2020; Toots et al., 2020). These results also suggest that molnupiravir-induced mutagenesis could be the molecular mechanism for its broad-spectrum anti-RNA virus activity. It is important to note that a study showed that molnupiravir could introduce mutations not only in the genome of SARS-CoV-2 but also in mammalian cells (Zhou et al., 2021). It is not clear whether it causes mutations in humans. In animal studies and clinical trials led by Merck, no mutagenic potential was found at normal doses in the duration of therapy. Therefore, the drug should be strictly administered as prescribed regarding dosing and contraindications such as pregnancy, and its risk should be evaluated over time.

AT-527, the hemisulfate form of AT-511, is an orally available purine nucleotide analog prodrug co-developed by Roche and Atea, which has pan-genotypic anti-HCV replication activity (Good et al., 2020). AT-527 is converted to a free base upon entry into cells and is subsequently metabolized to the triphosphate form metabolite AT-9010. AT-511 inhibited SARS-CoV-2 replication with an $\mathrm{EC}_{90}$ of $0.47 \mu \mathrm{M}$ in human respiratory epithelial cells, with similar activity against coronaviruses in Huh7 cells (Good et al., 2021). Of note, recent studies reported no anti-SARS-CoV-2 activities for AT511 in a variety of cell models, including Vero, Huh7, and human respiratory epithelial cells. The author suggests that differences between models may contribute to differences in the metabolism of AT-511 into the active form, thus compromising its antiviral activity (Do et al., 2021). Since AT-511 is a double prodrug requiring multiple enzymes for activation, it may be more susceptible to different experimental conditions (Do et al., 2021). To explore the mechanism of action of AT-527/AT-511 against SARS-CoV-2, Canard et al. (2021) determined the structure of the SARS-CoV-2 RdRp:RNA:AT-9010 complex via cryoelectron microscopy reconstruction and identified three sites of AT-9010 binding to NSP12. The compound is inserted into the nascent RNA strand at the activation site, and its 2'-methyl group blocks the accurate positioning of the second AT-9010 inserted into the following NTP site. Therefore, the incorporation of AT9010 terminates the RNA strand extension, and the subsequent AT-9010 disrupts the catalytic site of the complex, which exhibited resistance to ExoN removal. Besides, the 5'diphosphate of AT-9010 stably binds to the specific NSP12 N-terminal NiRAN domain of SARS-CoV-2, a structural and functional homolog of selenoprotein-O pseudokinase. This unique binding mode blocks the NiRANmediated UMPylation of SARS-CoV-2 NSP8 and NSP9 (Canard et al., 2021). The results indicate that AT-9010, the active form of AT-527, may exert anti-SARS-CoV-2 activity through a dual mechanism of inhibition via binding to both RdRp and NiRAN active sites. Atea evaluated AT-527 across multiple clinical trials, including the phase II MOONSONG virology study in patients with mild or moderate COVID-19, a global phase II study in hospitalized patients with moderate COVID-19, and the global phase III MORNINGSKY trial. On 19 October 2021, Atea Pharmaceuticals Inc. (2021) reported that the MOONSONG trial failed to meet its primary endpoint of reducing the viral load in patients treated with AT-527 relative to placebo in the overall population; however, it appeared to be effective in highrisk patients with underlying health conditions. The phase III MORNINGSKY trial (NCT04889040) is currently underway and will be completed in the second half of 2022 .

Galidesivir (BCX4430), an adenine analog originally developed by BioCryst to treat HCV, targets viral RdRp. Upon entry into cells, galidesivir is converted by host kinases to its triphosphate derivative, which is capable of mimicking ATP and is inserted by the RdRp complex into the newly synthesized RNA strand, inducing premature termination of nascent RNA (Taylor et al., 2016). Regarding the interaction of SARS-CoV-2 RdRp and galidesivir, docking analyses using the CCDC GOLD program demonstrated that galidesivir established six hydrogen and four hydrophobic bond interactions with NSP12, with an important one being the bond interaction with the catalytic center Asp ${ }^{760}$ or $\operatorname{Asp}^{761}$ (Zhang W. F. et al., 2020). In addition, interactions between galidesivir and $\mathrm{Asp}^{760} / \mathrm{Asp}^{761} / \mathrm{Asp}^{618}$ may interfere with the chelation of metal ions, thus affecting the activity of RdRp. Some studies have shown that galidesivir has broadspectrum antiviral activity against filoviruses, paramyxoviruses, coronaviruses, and SARS-CoV-2 in vitro, with an $\mathrm{EC}_{50}$ ranging from 3 to $68 \mu \mathrm{M}$ (Taylor et al., 2016; Dömling and Gao, 2020). A phase II clinical trial of galidesivir against COVID-19 is currently underway.

Sofosbuvir is a pyrimidine nucleotide analog approved by the FDA for the treatment of HCV. Sofosbuvir is effective against a variety of RNA viruses, including anti-SARS-CoV-2 activity in Huh7 cells, Calu-3 cells, and brain organoid models (Mesci et al., 2020; Sacramento et al., 2021). Several clinical trials on the efficacy of sofosbuvir/daclatasvir in patients with COVID-19 are underway in Iran (Sadeghi et al., 2020; Roozbeh et al., 2021). Sofosbuvir is converted to its active triphosphate form by cellular enzymes, and the active form is inserted into the newly synthesized RNA strand as a substrate of the SARS-Co-2 RdRp, immediately blocking the further extension of the RNA strand owing to the fluoro and methyl modifications at the 2' position, thereby inhibiting viral replication. Compared with natural nucleotide UMP excision, sofosbuvir terminated RNA was highly resistant to excision by the SARS-CoV-2 exonuclease complex (Jockusch et al., 2020b). 


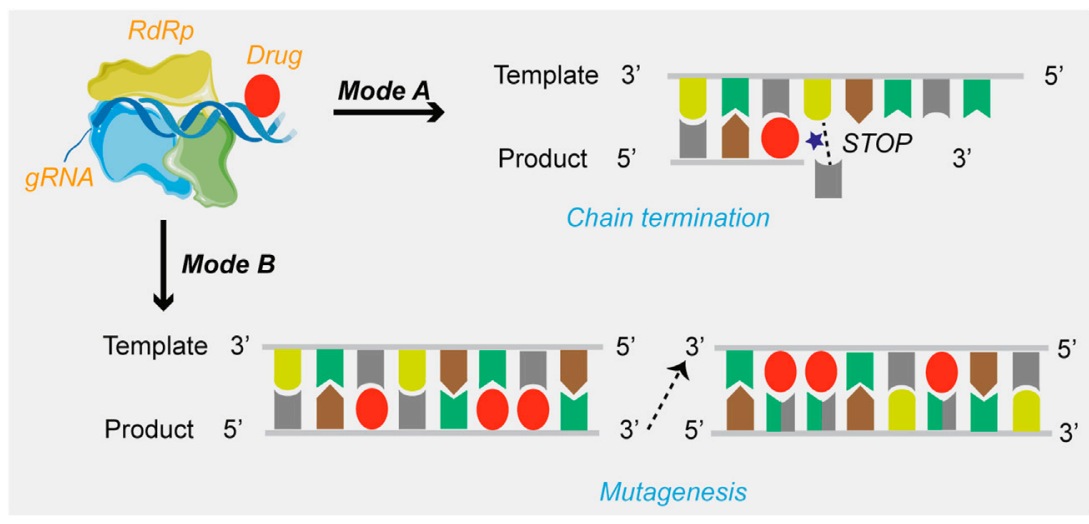

FIGURE 2 | The mechanisms of drugs targeting SARS-CoV-2 RdRp. Mode A: Chain termination; Mode B: Viral RNA mutagenesis induction.

Azvudine is a novel nucleoside analog that was initially developed for HIV treatment. It is the first nucleoside analog to simultaneously inhibit nucleoside reverse transcriptase and restore the expression of cytosine deaminase APOBEC3G in HIV-1 patient-derived CD4 ${ }^{+} \mathrm{T}$ cells (Yu and Chang, 2020). In July 2021, the drug was approved as a class 1 new drug to treat HIV infection. Currently, several clinical trials have been conducted on azvudine for COVID-19. Yu and Chang (2020) suggested that the mechanism of azvudine against SARS-CoV-2 may be similar to that of remdesivir. After conversion to triphosphate in vivo, $\mathrm{RdRp}$ incorporates it into the nascent RNA strand, preventing the subsequent addition of nucleotides. Nascent RNA synthesis is terminated, thereby inhibiting viral replication. The preliminary results of a clinical trial showed that azvudine treatment might shorten the nucleic acid negative conversion time in patients with mild or common COVID-19 (2.6 vs. 5.6 days, $p=0.008$ ) (Ren et al., 2020).

Tenofovir/Emtricitabine is an approved drug combination for the treatment and prevention of HIV infection, with more than nine clinical studies for treating COVID-19 currently registered on ClinicalTrials.gov. Mechanistically, tenofovir is phosphorylated to an active intracellular metabolite, and tenofovir triphosphate and emtricitabine triphosphate act on HIV reverse transcriptase, causing termination of viral DNA strand synthesis. The tenofovir/emtricitabine triphosphate binds tightly to SARS-CoV-2 RdRp and is incorporated as a substrate into the nascent RNA strand, resulting in the termination of strand synthesis (Jockusch et al., 2020a; Elfiky, 2020).

Currently, drug candidates targeting viral RdRp are the most dominant class in clinical trials for the treatment of COVID-19. Based on the review of the mechanisms of action, the mechanisms of SARS-CoV-2 RdRp complex inhibition can be grouped into two modes (Figure 2): immediate chain termination (e.g., remdesivir, galidesivir, sofosbuvir, and azvudine) and induced mutagenesis (e.g., favipiravir and molnupiravir). The difference in the mechanism may be attributed to differences in the structural complexity of the heterocycle of small molecules.

\subsubsection{Inhibitors of Viral Proteases-3CLpro}

PF-07321332 is an orally available inhibitor of 3CLpro, which plays a major role in the life cycle of coronaviruses. On 5 November 2021, Pfizer announced an interim analysis of phase II/III randomized, double-blind study of PAXLOVID ${ }^{\mathrm{TM}}$ in non-hospitalized adult patients with COVID-19, who are at high risk of progressing to severe illness. PAXLOVID ${ }^{\mathrm{TM}}$, a combination of PF-07321332 and ritonavir, significantly reduced hospitalization and death from any cause (Pfizer Inc., $2021)$. Further, $0.8 \%(3 / 389)$ of patients were admitted to the hospital through day 28 with no deaths in the PAXLOVID ${ }^{\mathrm{TM}}$ group, compared to $7.0 \%$ (27/385) of patients were admitted, with seven subsequent deaths in the placebo group. An $89 \%$ reduction in the risk of COVID-19 hospitalization or death was reported in patients with PAXLOVID ${ }^{\mathrm{TM}}$ treatment within 3 days of symptom onset $(p<0.0001)$. Regarding safety, 19 and $21 \%$ of patients experienced adverse events in the PAXLOVID ${ }^{\mathrm{TM}}$ and placebo group, respectively, with the former being less likely to report serious adverse events (1.7 vs. 6.6\%) (Mahase, 2021). Pfizer will cease further enrollment and plans to submit the data to the FDA for Emergency Use Authorization (EUA). As a 3CLpro inhibitor, PF-07321332 reacts with a cysteine residue at the proteasebinding site to prevent viral replication in cells. This cysteine plays a key role in the 3CLpro activity of coronaviruses. Therefore, activity inhibition prevents the viral genome from encoding functional NSPs, ultimately suppressing viral replication (Muramatsu et al., 2016; Ionescu, 2020). PF07321332, which contains a nitrile group, is a polypeptide covalent inhibitor. As reversible site-specific inhibitors, peptide nitriles react with catalytic cysteine to form sulfides. The inhibitor presents a g-lactam ring, a common feature of most covalent inhibitors developed against SARS-CoV-2 3CLpro, which exploits the selectivity of glutamine residues at cleaved multiprotein equivalent sites (Ramos-Guzmán et al., 2021). Ramos-Guzmán et al. revealed some critical characteristics of PF-07321332 using computational simulations. The inhibitor acts in two stages: forming a non-valent complex with the enzyme and a reaction with the catalytic cysteine to form sulfide (RamosGuzmán et al., 2021). With regard to the binding process, 
molecular dynamics simulations and free energy calculations indicated that most of the affinity between the inhibitor and the enzyme was due to the P1 and P2 groups, which are identical or similar to most known inhibitors of this protease; While the P3 and $\mathrm{P} 4$ groups also contribute to the binding process, the free energy contribution is much smaller, suggesting that it is still possible to improve the affinity of 3CLpro (Ramos-Guzmán et al., 2021). The low molecular weight of the nitrile group appears to be superior to covalent inhibition. The resulting sulfide is steadier than the initial non-covalent complex, indicating that the inhibition is more irreversible compared to that of aldehydes or ketones derivatives.

PF-07304814, an early intravenously administered drug developed by Pfizer, is also a 3CLpro inhibitor and is hydrolyzed into its active form, PF-00835231. Recent studies have shown that PF-07304814 also has inhibitory activity against the 3CLpro of SARS-COV-2. In Vero E6 cells, the EC E0 $_{50}$ PF07304814 against SARS-CoV-2 was $40 \mu \mathrm{M}$, and, after the addition of p-glycoprotein, the $\mathrm{EC}_{50}$ was $0.48 \mu \mathrm{M}$ (Boras et al., 2021). Currently, the drug is in phase I clinical trials (NCT04627532 and NCT04535167) for COVID-19 treatment.

S-217622 is a small-molecule anti-SARS-CoV-2 candidate drug developed by Shionogi, Japan, which inhibits 3CLpro activity to prevent viral infection. S-217622 exhibited in vitro antiviral activity against multiple SARS-CoV-2 strains, including alpha, beta, gamma, and delta. In an animal model, the viral load was rapidly and significantly reduced after administration (Shionogi \& Co., Ltd., 2021a). Phase I clinical trials conducted in July 2021 in Japan showed that oral administration of S-217622 was safe, with once-daily oral dosing reducing plasma concentrations, as predicted from preclinical studies (Shionogi \& Co., Ltd., 2021c). Phase II/III clinical trials for patients with mild COVID-19 or asymptomatic infection are currently underway (Shionogi \& Co., Ltd., 2021b).

FB2001 is a protease inhibitor developed by the Shanghai Institute of Materia Medica, Chinese Academy of Sciences. The antiviral drug was designed and synthesized based on the three-dimensional structure of SARS-CoV-2 3CLpro with the aim of effectively blocking viral replication and inhibiting progeny virions production. At the cellular level, the $\mathrm{EC}_{50}$ of FB2001 against SARS-CoV-2 was $0.53 \mu \mathrm{M}$, and it showed better pharmacokinetic properties and safety in rat and beagle dog models (Dai et al., 2020). In terms of its inhibitory mechanism on 3CLpro of SARS-CoV-2, a structure-based study revealed the FB2001-3CLpro binding mode. FB2001 occupied the substrate-binding pocket and further inhibited 3CLpro activity. The catalytic site Cys ${ }^{145}$ of 3CLpro forms a covalent bond with the carbon atom of the aldehyde group of FB2001, and the Oxygen atom of the aldehyde group stabilizes the compound conformation by forming a hydrogen bond with $\mathrm{Cys}^{145}$ at the $\mathrm{S1}^{\prime}$ site. The oxygen atom of the lactam group forms a hydrogen bond with the side chain of His ${ }^{163}$, the NH group also forms hydrogen bonds with $\mathrm{Phe}^{140}$ and Glu ${ }^{166}$, stabilizing the lactam group. In addition, the cyclohexyl group of the FB2001 interacts with the side chains of residues Asp ${ }^{187}$, $\mathrm{Arg}^{188}, \mathrm{Met}^{49}$, $\mathrm{Met}^{165}$, and $\mathrm{Tyr}^{54}$ through hydrophobic interactions, and the indole group interacts with residue $\operatorname{Gln}^{189}$ and Pro ${ }^{168}$ in the same way (Dai et al., 2020). FB2001 has been initiated in a phase I/II clinical trial (NCT04766931) in the United States in March 2021 to evaluate the safety and efficacy of FB2001 for the treatment of COVID-19.

Ebselen is a small-molecule organoselenium compound with glutathione peroxidase activity. The $\mathrm{IC}_{50}$ of its anti-SARS-CoV-2 activity in Vero cells was $4.67 \mu \mathrm{M}$, and the $\mathrm{EC}_{50}$ of its hydrolytic activity against $3 \mathrm{CL}$ pro of SARS-CoV-2 was $0.67 \mu \mathrm{M}$, suggesting that ebselen may exert antiviral effects by inhibiting SARS-CoV-2 3CLpro (Jin et al., 2020). Amporndanai et al. investigated the binding mode of 3CLpro and ebselen via high-resolution cocrystallography. They found that the formation of a selenyl sulfide bond between Cys ${ }^{145}$ of the catalytic dyad and the free selenium atom at the catalytic site inhibited the activity of 3CLpro. Further, this binding did not impact the conformational changes of amino acid disability around the active site. Combined with the LC/MS method, it was shown that after ebselen hydrolysis, the selenium atom binds directly to the catalytic site $\mathrm{Cys}^{145}$ to exert antiviral effects (Amporndanai et al., 2021). Phase II clinical trials of ebselen for treating patients with moderate to severe COVID19 are currently ongoing.

Both ritonavir and lopinavir are HIV protease inhibitors. Ritonavir inhibits aspartic protease, which prevents processing of the Gag-Pol polyprotein precursor, and lopinavir inhibits GagPol polyprotein division. They act synergistically to reduce the viral load by disabling the regeneration of viral particles (Cvetkovic and Goa, 2003). They are likely to exert antiviral activity by inhibiting 3CLpro of coronaviruses, including SARSCoV-2 (Wu et al., 2004; Uzunova et al., 2020). A clinical study showed that lopinavir/ritonavir was less effective than favipiravir in terms of viral clearance and disease progression, with more adverse events recorded (Cai et al., 2020). In addition, several clinical trials, including Solidarity conducted by the WHO, indicated that lopinavir/ritonavir did not significantly reduce mortality and clinical symptoms of patients with COVID-19 (Cao et al., 2020; Pan et al., 2021).

Triazavirin is a guanine nucleotide analog that was originally approved for the treatment of influenza in Russia. Triazavirin has broad-spectrum antiviral activity against RSV, influenza, parainfluenza virus, and adenovirus, but its antiviral mechanism remains unclear. It is widely speculated to act as a purine nucleotide analog inhibiting viral RNA synthesis and replication of viral genome fragments (Wu X. et al., 2020). A computational docking study speculated that triazavirin might exert anti-SARS-CoV-2 effects as a 3CLpro inhibitor (Shahab and Sheikhi, 2020). Preliminary results from a randomized controlled trial of triazavirin for COVID-19 showed no significant benefit for patients with COVID-19. However, patients in this arm less frequently used concomitant therapies for hepatic, respiratory, cardiac, or renal conditions (Wu X. et al., 2020). Two clinical trials of triazavirin for the treatment of COVID-19 are ongoing in South Africa and Egypt.

3CLpro is an ideal anti-SARS-CoV-2 target along with the classical target, RdRp. Drug repurposing screening and structure-based design of novel drug candidates targeting 


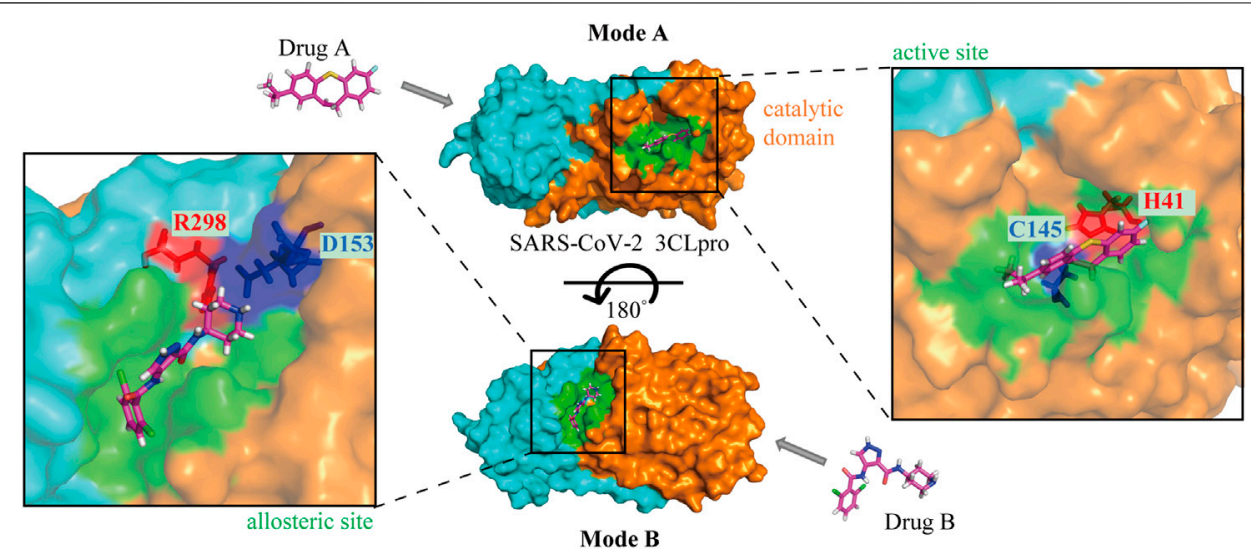

FIGURE 3 | The mechanisms of drugs targeting SARS-CoV-2 3CLpro. Mode A: Binding to the active site of the catalytic domain; Mode B: Binding to the allosteric site. The orange and aqua areas represent the catalytic and dimerization domains, respectively. Drug A in the $3 C L$ pro active site with catalytic residues labeled, and drug $\mathrm{B}$ in the allosteric site with residues labeled.

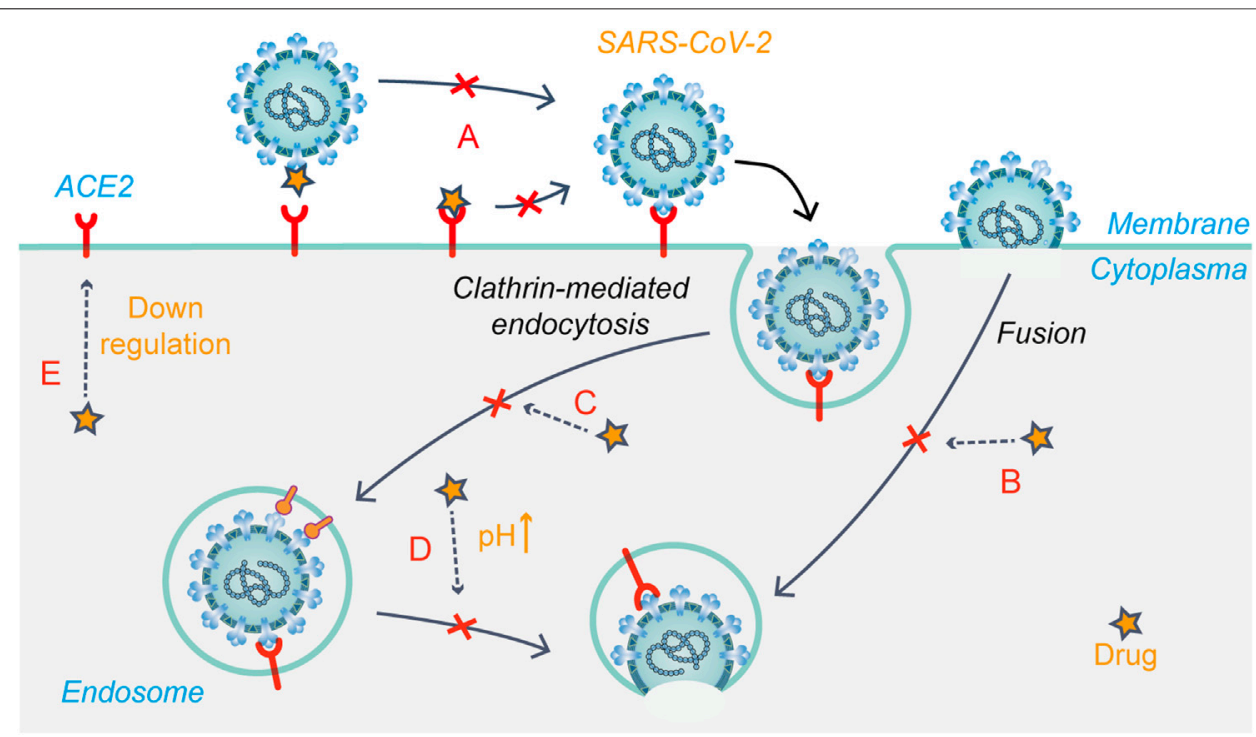

FIGURE 4 | Schematic presentation of the possible mechanism that drug candidates target at early stages of SARS-CoV-2 life cycle. (A) Modification of S glycoprotein and the ACE2 receptor glycoprotein; (B) Fusion and release (C) Endocytosis-mediated viral entry; (D) Changing the acidic environment; (E) ACE2 receptor regulation.

3CLpro have become hot spots for anti-SARS-CoV-2 drug development since the cryo-electron microscopy structure has been reported. In general, the enzymatic activity of 3CLpro is dependent on the structure of the catalytic site, so the majority of 3CLpro inhibitors hinder the activity by affecting the dimerization of the enzyme and altering the structure of the catalytic active site in the substrate-binding pocket, especially the residue $\mathrm{Cys}^{145}$ at the S1' site (Figure 3). In addition to the catalytic active site, the allosteric site may also be a binding region for drug interaction (Figure 3), although fewer compounds have been identified to act at this site. Further study on the key allosteric sites for enzyme inhibition may improve the efficiency of novel drug development.

\subsection{Antiviral Agents Targeting Host Factors 4.2.1 Inhibitors of Endosomal Entry}

Chloroquine was originally used to treat malaria, but its use declined with the emergence of drug-resistant malaria parasites. Chloroquine and hydroxychloroquine are also used for the treatment of rheumatic diseases because of their immunomodulatory effects. They have good inhibitory effects on a variety of coronaviruses, including SARS-CoV-2 (Rolain et al., 2007; Wang M. et al., 2020; Persoons et al., 2021). Based on relevant reports, the mechanism of action of chloroquine/ hydroxychloroquine against coronavirus mainly involves the modification of viral S glycoprotein and post-translational modification of the ACE2 receptor glycoprotein ( $\mathrm{Li}$ et al., 
2003; Vincent et al., 2005). Chloroquine/hydroxychloroquine may inhibit SARS-CoV-2 replication through a variety of mechanisms, acting on both the virus and host cells. Studies have shown that chloroquine/hydroxychloroquine not only inhibits the endocytosis-mediated viral entry (Figures 4A, C) into host cells through its effect on endosome maturation but also affects the post-entry stage (Liu et al., 2020). Chloroquine phosphate effectively inhibited SARS-CoV-2 infection in vitro, shortened the time of viral clearance, and improved clinical symptoms in patients with COVID-19 (Huang et al., 2020). Early into the COVID-19 outbreak, the FDA granted chloroquine emergency authorization for the treatment of COVID-19. Since then, several clinical trials have shown that hydroxychloroquine is ineffective in COVID-19 and has serious side effects (Geleris et al., 2020; National Institutes of Health, 2020). Further, researchers negated the anti-SARS-CoV-2 activity of hydroxychloroquine in a human lung cell model (Hoffmann et al., 2020b). At present, hydroxychloroquine is considered ineffective against COVID-19, while the efficacy of chloroquine remains controversial.

Arbidol (umifenovir) could effectively inhibit viral fusion with host cells and has been approved to prevent and treat influenza in Russia. Previous studies have shown that arbidol effectively inhibits SARS-CoV-2 infection in vitro with an $\mathrm{EC}_{50}$ value of $4.11 \mu \mathrm{M}$. This study further investigated the action stages of arbidol on the virus life cycle, showing that the drug blocks SARS-CoV-2 entry by preventing attachment and release from intracellular vesicles (Wang X. et al., 2020). Molecular dynamics and structural studies suggested that there may be structural similarities between the drug-binding regions of influenza virus HA and SARS-CoV-2 S glycoprotein. Thus, arbidol may target the latter and impede its trimerization to exert antiviral effects (Vankadari, 2020) (Figures 4A,B). A meta-analysis showed that arbidol use was not related to symptom alleviation, nucleic acid negative conversion time, or mortality in COVID-19 patients compared to the control treatment (Huang et al., 2021). There is no clear evidence to support the efficacy of arbidol in treating COVID-19.

Niclosamide is an FDA-approved anthelmintic drug, which showed high anti-SARS-CoV-2 activity in Vero E6 cells and respiratory tract infection models (Jeon et al., 2020). The antiSARS-CoV-2 mechanism of the drug is currently unclear. Niclosamide reportedly reduces receptor-binding domain and dextran uptake while increasing that of transferrin, resulting in an enhanced density of transferrin endosomes (Prabhakara et al., 2021). In addition, the $\mathrm{pH}$ of both early and late endosomes changed from acidic to neutral after niclosamide treatment. These phenomena suggest that niclosamide inhibits viral entry into cells by affecting $\mathrm{pH}$ dependent endocytosis pathways to hinder viral internalization (Prabhakara et al., 2021) (Figure 4D). A phase I trial showed that intranasal and inhalation administration of niclosamide exhibited dose-dependent pharmacokinetics, and was well-tolerated in healthy volunteers (Backer et al., 2021). Niclosamide add-on therapy was beneficial for reducing the time needed for recovery ( 5 vs. 7 days, $p=0.005$ ), but it did not reduce mortality from SARS-CoV-2 infection (Abdulamir et al., 2021). Currently, eight relevant clinical trials on niclosamide have been registered.

Chlorpromazine is an FDA-approved drug for treating psychiatric disorders, nausea and vomiting during pregnancy, and cancer. In vitro experiments have shown that chlorpromazine has broad-spectrum antiviral activity. In Vero E6 and A549-ACE2 cell models, the $\mathrm{IC}_{50}$ value of chlorpromazine against SARS-CoV-2 was about $9-10 \mu \mathrm{M}$ (Plaze et al., 2021). Inoue et al. found that chlorpromazine impedes the clathrin-mediated endocytosis pathway by inhibiting the relocalization of clathrin and adaptor protein 2 on the cell surface (Figure 4C), thus inhibiting SARS-CoV entry (Inoue et al., 2007). In addition, studies have shown that chlorpromazine has immunomodulatory effects and exerts antiviral effects by increasing IgM levels in the blood (Plaze et al., 2020). Therefore, chlorpromazine is likely to inhibit SARS-CoV-2 infection through the mechanisms mentioned above. Currently, there are two clinical trials registered for chlorpromazine to treat COVID-19, but recruitment has not yet been conducted.

Baricitinib is an oral JAK1/JAK2 inhibitor. In November 2020, it was authorized for emergency use in combination with remdesivir for the treatment of hospitalized COVID-19 patients requiring supplemental oxygen, non-invasive/invasive mechanical ventilation, or ECMO. Zhang X. et al. (2020) reported a possible mechanism of baricitinib against SARS-CoV-2. AAK1 and GAK are two key regulators of the ACE2 receptor, mediating clathrin-dependent endocytosis (Lu et al., 2020) (Figures 4C, E). Baricitinib has a high binding affinity for AAK1 and GAK, blocking SARS-CoV-2 entry into cells for replication and intracellular assembly. Baricitinib, on the other hand, is an ATP-competitive kinase inhibitor that selectively and effectively inhibits JAK1/JAK2. By inhibiting JAK1/JAK2, baricitinib inhibits the pro-inflammatory signals of various cytokines, such as IL-6, IL-12, and IL-23 (Keystone et al., 2015). In severe cases of COVID-19, the JAK-STAT signaling pathway is also involved in cytokine storms, which baricitinib may help suppress, thus preventing damage to the lungs and other organs (Zhang X. et al., 2020). In a randomized controlled trial, baricitinib plus standard of care treatment was associated with reduced mortality in hospitalized COVID-19 patients, and the 28 -day all-cause mortality was $8 \%(n=62)$ for baricitinib and $13 \%(n=100)$ for placebo [HR 0.57 (95\% CI 0.41-0.78); $p=$ 0.0018] (Marconi et al., 2021).

\subsubsection{Inhibitors of TMPRSS2}

Proxalutamide is an androgen receptor antagonist developed by Kintor Pharmaceutical Limited and was initially used to treat prostate cancer. According to related studies by Kintor, proxalutamide blocks the SARS-CoV-2 entry by inhibiting androgen receptor function and downregulating ACE2 and TMPRSS2 expression at the transcriptional level (Wu S. et al., 2020). ACE2 facilitates viral entry into host cells by binding to the S1 domain of the S protein. TMPRSS2 is located on the surface of host cells and is responsible for cleavage of viral $S$ protein and ACE2, impeding subsequent membrane fusion during coronavirus entry. Activated androgen receptors induce the 
expression of TMPRSS2. Phase I/II clinical studies of proxalutamide for COVID-19 treatment have been completed, showing that proxalutamide can effectively accelerate viral clearance and reduce the time of clinical symptom alleviation in patients with mild to moderate COVID-19 (Cadegiani et al., 2021). However, the excellent trial data has also raised some questions about this anti-SARS-CoV-2 drug, plunging the drug into controversy. The most effective way to address the controversy is to obtain data from rigorous clinical trials. Several phase III clinical trials are currently underway in the United States and Brazil. The update of the phase III study (NCT04870606) of proxalutamide for COVID-19 was provided by Kintor Pharma, and the interim analysis of 348 patients enrolled in the trial showed that it did not meet the statistical criteria due to the small number of events (Kintor Pharma-B, 2021).

Dutasteride is a 5-alpha-reductase inhibitor that specifically binds to isoenzymes $1 / 2$ of 5 -alpha reductase to form a stable enzyme complex and inhibits the conversion of testosterone to 5alpha-dihydrotestosterone. Dihydrotestosterone stimulates androgen receptor activity, and the activated androgen receptor regulates TMPRSS2 expression (Mollica et al., 2020). Therefore, it is likely that dutasteride affects viral entry into cells by reducing TMPRSS2 expression, and thus inhibits SARS-CoV2 infection.

Camostat mesylate and nafamostat mesylate are structurally similar guanidinobenzoic acid derivatives. As cellular protease inhibitors, they can effectively suppress the activities of kallikrein, trypsin, thrombin, and TMPRSS2. Studies have shown that TMPRSS2 is a type II transmembrane serum protease widely expressed in epithelial cells that have been shown to facilitate the entry of a variety of viruses into cells, including influenza, SARS$\mathrm{CoV}$, and MERS-CoV (Breining et al., 2021). The TMPRSS2 inhibitors nafamostat mesylate and camostat mesylate effectively suppress SARS-CoV-2 activity in vitro (Hoffmann et al., 2020a; Wang M. et al., 2020). Several clinical trials of camostat mesylate for the treatment of COVID-19 have been carried out. The results of one randomized controlled trial showed no improvement in clinical symptoms and mortality in hospitalized COVID-19 patients (Gunst et al., 2021). A phase II clinical trial (NCT04623021) of nafamostat mesylate has been completed; however, the results are not yet available, and the phase III clinical trial (NCT04871646) for COVID-19 was conducted in South Korea in June. Camostat mesylate is rapidly hydrolyzed in vivo to its active metabolite 4-(4guanidinobenzoyloxy) phenylacetic acid, with similar antiSARS-CoV-2 activity (Hoffmann et al., 2021). Relevant model prediction studies have shown that camostat mesylate binds to all three pockets of TMPRSS2 (S1, catalytic triad domain, and hydrophobic patch). In contrast, bromhexine, another TMPRSS2 inhibitor, only binds to the hydrophobic patch domain (Breining et al., 2021). Furthermore, the hydrogen bond and van der Waals interaction with $\mathrm{Asp}^{435}$ of S1, Ser ${ }^{441}$ of the catalytic domain, and $\mathrm{His}^{296}$ of the hydrophobic patch were predicted. However, a report suggests that the anti-SARS-CoV-2 activity of camostat mesylate and its metabolites may not only result from the competitive inhibition of TMPRSS2 since covalent inhibition as substrates may also contribute to antiviral activity (Sun et al., 2020). Therefore, the molecular mechanism of camostat mesylate or GBPA on TMPRSS2 requires further study.

\subsubsection{Inhibitors of Dihydroorotate Dehydrogenase}

Both PTC299 and teriflunomide are inhibitors of DHODH, a key enzyme in the pyrimidine de novo biosynthesis pathway, which SARS-CoV-2 can hijack for virus replication. DHODH inhibitors reportedly suppress the production of virusinduced inflammatory cytokines by regulating lymphocyte activation, exerting dual antiviral and anti-inflammatory effects (Xiong et al., 2020). In tissue culture, PTC299 exhibited potent anti-SARS-CoV-2 replication activity $\left(\mathrm{EC}_{50}\right.$ of $\left.2.0-31.6 \mu \mathrm{M}\right)$. Consistent with reported DHODH requirements for inflammatory cytokine production, PTC299 effectively inhibited the production of IL-6, IL-17A, IL-17, and VEGF in tissue culture models (Luban et al., 2021). In Vero E6 cells, teriflunomide exhibited potent antiviral effects, with an $\mathrm{EC}_{50}$ of $26.06 \mu \mathrm{M}$. Similarly, teriflunomide and its active form leflunomide also exerted antiviral effects by limiting pyrimidine de novo synthesis and immunomodulatory effects (Xiong et al., 2020). Currently, there are three clinical trials of leflunomide against COVID19 registered on ClinicalTrials.gov, as well as one clinical trial registration for PTC299 against COVID-19.

BAY 2402234 is a novel antitumor drug developed by Bayer, currently undergoing phase I clinical trials for myeloid malignancies. With regard to anti-SARS-CoV-2, various DHODH inhibitors have shown significant antiviral efficacy, especially BAY 2402234, which is currently the most effective small-molecule agent against SARS-CoV-2. BAY 2402234 can effectively reduce viral RNA load, with an $\mathrm{IC}_{50}$ of $7.0 \mathrm{nM}$ in Vero E6 cells (Song, 2020). It has a good therapeutic potential for the treatment of SARS-CoV-2. The crystal structure of the DHODH protein with BAY 2402234 shows that the latter binds to the ubiquitin-binding site between the $\mathrm{N}$-terminal helices, and predicts a non-classical hydrogen bond between the hydroxyl of $\mathrm{Thr}^{63}$ and the fluorine (hydrogen bond acceptor) of the terminal phenyl group (Christian et al., 2019). The triazolone group binds to the deep hydrophilic end of the pocket via hydrogen bonding with $\mathrm{Gln}^{47}$ andTyr ${ }^{356}$, the hydroxyl group interacts with $\mathrm{Thr}^{360}$ via a bound water molecule, and the compound has extensive hydrophobic interactions with several non-polar residues at the entrance of the binding site (Christian et al., 2019).

\subsubsection{Inhibitors of Selective Serotonin Reuptake}

Fluvoxamine is a selective serotonin reuptake inhibitor that is FDA approved for the treatment of obsessive-compulsive disorder and depression. Several phase II clinical trials of fluvoxamine against COVID-19 have been registered. The TOGETHER randomized clinical trial (NCT04727424) was conducted to evaluate the effect of early fluvoxamine 
treatment and the results were published recently. In a perprotocol analysis of patients, acute treatment with fluvoxamine reduced the need for hospitalization among high-risk outpatients (RR 0.34, 95\% BCI, 0.21-0.45) (Reis et al., 2022). Previous studies have shown that fluvoxamine has anti-inflammatory effects. In immune cells, fluvoxamine binds to S1R, leading to reduced production of immunomodulatory cytokines. In human endothelial cells, it reduces the expression level of inflammation-related genes (Rafiee et al., 2016; Rosen et al., 2019). Lenze et al. (2020) proposed that fluvoxamine may exert antiviral effects by affecting the S1R-IRE1 pathway or through its lysosomotropic properties. Kornhuber et al. (2021) showed that acid sphingomyelinase cleaves sphingomyelin into lipophilic ceramide, forming large gel-like rafts on the membrane, which SARS-CoV-2 can use to enter cells. Thus, fluvoxamine may exert its anti-SARS-CoV-2 effects by inhibiting acid sphingomyelinase.

\subsubsection{Inhibitors of eEF1A}

Plitidepsin is a cyclic depsipeptide approved for the treatment of patients with refractory multiple myeloma in Australia. Several clinical trials have investigated its efficacy and safety as a candidate drug for COVID-19 treatment. White et al. (2021) showed that plitidepsin displays high anti-SARS-CoV-2 activity, with an $\mathrm{IC}_{50}$ value of $0.88 \mathrm{nM}$ in a hACE2-HEK293T cell model, and the antiviral activity was achieved through eEF1A activity inhibition. eEF1A is involved in the enzymatic delivery of aminoacyl tRNA to ribosomes and aminoacylation-dependent tRNA export pathways during the mRNA translation of RNA viruses. Plitidepsin suppresses the translation of SARS-CoV-2 ORF1A and ORF1B by acting on eEF1A, resulting in reduced expression levels of polyproteins $1 \mathrm{a}$ and $\mathrm{b}$, further decreasing the expression of NSPs such as RdRp (Wong and Damania, 2021). In addition, the drug inhibits the translation of subgenomic mRNAs, also leading to reduced expression levels of viral structural and auxiliary proteins.

\subsubsection{Inhibitors of IMP $\alpha / \beta 1$}

Ivermectin is an FDA-approved antiparasitic agent with broadspectrum antiviral activity. Ivermectin effectively inhibited SARSCoV-2 infection in Vero-hSLAM cells (Frieman et al., 2007). However, the exact mechanism of action of the drug against SARS-CoV-2 is unclear. It may block the transport of viral proteins into the nucleus by targeting host IMP $\alpha / \beta 1$, thereby suppressing viral replication (Yang et al., 2020). Similarly, during SARS-CoV infection, IMP $\alpha / \beta 1$ has a potential role in the signaldependent nucleocytoplasmic shutting of the virus nucleocapsid protein, potentially impacting host cell division (Caly et al., 2020). Besides, the viral ORF6 antagonizes the antiviral activity of the STAT1 transcription factor by sequestering IMP $\alpha / \beta 1$ on the ER/ Golgi membrane (Frieman et al., 2007). In summary, ivermectin may exert anti-SARS-CoV-2 effects by inhibiting nuclear transport activity. To date, more than 60 registered clinical trials related to ivermectin for the treatment of COVID-19, only a few of which are randomized controlled trials, have reported mortality. Based on reported results, there is insufficient evidence on the efficacy of ivermectin against
COVID-19; thus, the WHO does not recommend its use, except in clinical trials (Yavuz and Komsuoğlu Çelikyurt, 2021).

\subsubsection{Inhibitors of Phosphoinositide Kinase, FYVE-Type Zinc Finger Containing (PIKfyve)}

Apilimod is a small-molecule inhibitor of PIKfyve. Apilimod was reported to have high anti-SARS-CoV-2 activity in A549-ACE2 and Vero cells, with $\mathrm{IC}_{50}$ values of $0.007 \mu \mathrm{M}$ and $<0.08 \mu \mathrm{M}$, respectively. The authors proposed that the antiviral activity could be ascribed to the inhibition of PIKfyve phosphorylation in SARS-CoV-2 infection (Bouhaddou et al., 2020). Bouhaddou et al. (2020) reported that PIKfyve is one of the major enzymes involved in PI $(3,5)$ P2 synthesis during early endocytosis. Entry inhibition assay showed that apilimod effectively blocked the entry of SARS-CoV-2 pseudovirus particles into HEK293-hACE2 cells by inhibiting PIKfyve activity, suggesting that PIKfyve may be a potential target for viral entry into cells via endocytosis, and apilimod inhibits SARS-CoV-2 infection through this target. Kang et al. (2020) also suggested that apilimod interfered with endosome transport and prevented virus entry by inhibiting PIKfyve activity. However, it should be noted that apilimod also selectively inhibits IL-12 and IL-23 by inhibiting the phosphotransferase activity of PIKfyve (Cai et al., 2013). It has been reported that PIKfyve is an enzyme required for MHC class II trafficking to the cell surface, and apilimod-mediated inhibition of this process may reduce the expression of MHC class II and type I interferon. A similar phenomenon is observed during SARS-CoV-2 infection. Therefore, the use of apilimod may suppress the immune system in patients with COVID-19 (Baranov et al., 2020). AI Therapeutics, Inc. is conducting a phase II clinical trial (NCT04446377) to evaluate the efficacy of apilimod in patients with COVID-19.

\subsubsection{Antiviral Agents Targeting Multiple Life Cycle Stages}

Nitazoxanide, a thiazolide drug approved by the FDA to treat protozoal and helminthic infections, exhibited broad-spectrum antiviral activity in vitro (Mahmoud et al., 2020). In the Vero E6 cell model, the $\mathrm{EC}_{50}$ value of nitazoxanide against SARS-CoV-2 was $2.12 \mu \mathrm{M}$ (Wang M. et al., 2020). Regarding its antiviral mechanism of action, nitazoxanide may target multiple stages of the SARS-CoV-2 life cycle, including endocytosis and membrane fusion, viral genome synthesis, viral release, and the inflammatory response (Lokhande and Devarajan, 2021). Currently, there are about 30 related clinical trials registered on ClinicalTrials.gov. The results of a pilot trial (NCT04348409) showed that nitazoxanide treatment shortened hospitalization time (6.6 vs. 14.0 days, $p=0.021$ ), accelerated viral clearance, and reduced inflammatory cytokines (Blum et al., 2021). A metaanalysis of randomized controlled trials showed that nitazoxanide could improve viral elimination (Zhang et al., 2021).

\section{DISCUSSION}

As the COVID-19 pandemic continues, the research and development of anti-SARS-CoV-2 drugs remain of utmost importance. The drug discovery strategy applied in the early 
stages of the COVID-19 outbreak was an extensive screening of available agents for drug repurposing. With the subsequent and still ongoing elucidation of the function and structure of SARSCoV-2 proteins, the screening of small molecules based on their targets will represent a more effective strategy to discover effective drugs against SARS-COV-2.

Targets include viral and host proteins involved with the viral life cycle. Although there are various therapeutic targets for the treatment against SARS-CoV-2, the major focus of small-molecule drug development is on RdRp and 3CLpro. RdRp acts as a crucial component in SARS-CoV-2 replication, and RdRp inhibitors have shown good efficacy against SARS-CoV-2 in vitro as well as in clinical trials, with examples including molnupiravir, AT-527, and favipiravir. 3CLpro, another enzyme essential for viral replication, is the main protease of SARS-CoV-2. Since there is no protease with a similar cleavage site to 3CLpro in humans, it is more likely to develop highly specific inhibitors with good safety and broadspectrum anti-coronavirus activity, such as PF-07321332 and s217622. There are relatively few studies on the anti-SARS-CoV-2 mechanism of action of drugs against host targets, probably because host intracellular pathways are complex and the mechanisms vary between indications, limiting the available relevant mechanistic studies. The current host targets of antiSARS-CoV-2 clinical drugs mainly involve the factors from the viral endocytosis pathway, intracellular kinases (e.g., TMPRRS2, DHODH, PIKfyve), and cellular immune regulators. Considering that these targets exert multiple functions within the cell, drugs targeting them should be further characterized with respect to toxicity and timing of administration.

The elucidation of mechanisms may provide a theoretical basis for drug combinations. The widespread use of drugs usually leads to the development of drug resistance as a result of natural selection, and with the successive approval and widespread use of anti-SARS-CoV-2 small-molecule drugs, the emergence of drugresistant strains will be one of the key concerns. One of the main strategies utilized for combating drug resistance is drug combinations, especially those with different mechanisms of action. Combining of drugs, such as molnupiravir and PF07321332, to inhibit SARS-CoV-2 replication may theoretically lead to stronger effects and reduce the development of drug resistance. Indeed, whether this leads to better results needs to be verified in clinical trials. At the same time, whether the combination of drugs leads to stronger side effects is an issue that needs to be considered.

\section{REFERENCES}

Abdulamir, A. S., Gorial, F. I., Saadi, S. J., Maulood, M. F., Hashim, H. A., Alnuaimi, A. S., et al. (2021). A Randomised Controlled Trial of Effectiveness and Safety of Niclosamide as Add on Therapy to the Standard of Care Measures in COVID-19 Management. Ann. Med. Surg., 69 102779. doi:10. 1016/j.amsu.2021.102779

Amporndanai, K., Meng, X., Shang, W., Jin, Z., Rogers, M., Zhao, Y., et al. (2021). Inhibition Mechanism of SARS-CoV-2 Main Protease by Ebselen and its Derivatives. Nat. Commun. 12 (1), 3061. doi:10.1038/s41467-021-23313-7

Appili Therapeutics Inc. (2020). Appili Therapeutics Announces Last Patient Enrolled in Phase 3 Trial of Oral Avigan ${ }^{\circledR} /$ Reeqonus $^{\mathrm{TM}}$ for the Treatment of Mild-To-
A key factor in efficacy of oral antivirals is administering early in the patient's infection. This was the case in the clinical trials of PAXLOVID and molnupiravir, where the treatments were initiated within 3 and 5 days of symptom onset, respectively. Moreover, clinical studies of favipiravir for COVID-19 have also demonstrated better outcomes with early rather than late dosing in Japan (Shinkai et al., 2021). Another concern is whether the efficacy of oral antiviral drugs is compromised in patients who have developed breakthrough infections after vaccination. It's obvious that the emergence of Omicron, a new SARS-CoV-2 variant, has increased the risk of breakthrough infections. To our knowledge, the clinical efficacy of both PAXLOVID and molnupiravir have not been evaluated in clinical trials in vaccinated populations. A phase III clinical trial of favipiravir for COVID-19 showed that the median time to meet the primary endpoint was 2.9 days shorter in the subgroup of patients with IgA or IgG antibodypositive compared to the subgroup of patients with IgA or IgG antibody-negative (Shinkai et al., 2021). This indicates that oral antivirals may also show better efficacy in patients with breakthrough infections.

Discovering and clarifying the mechanism of action for candidate anti-SARS-CoV-2 drugs is a key point in developing broad-spectrum, target-specific drugs. The advance of structural biology, artificial intelligence, and bioinformatics has provided important tools for studying the mechanism of action and accelerating the development of antiviral drugs. Elucidating the mechanism of drug activity provides a theoretical basis for the development of highly effective and specific drugs, drug combinations, and multi-target drug development for the treatment of COVID-19.

\section{AUTHOR CONTRIBUTIONS}

LZ and WZ contributed to all aspects of the final work. SL conceptualized the research work and reviewed the manuscript.

\section{FUNDING}

This work was supported by the National Science and Technology Major Projects "Major New Drugs Innovation and Development" (2018ZX09711003).

Moderate COVID-19. Available at: https://www.appilitherapeutics.com/newsfeed/ Appili-Therapeutics-Announces-Last-Patient-Enrolled-in-Phase-3-Trial-of-OralAvigan\%C2\%AE\%2FReeqonus\%E2\%84\%A2-for-the-Treatment-of-Mild-toModerate-COVID-19 (Accessed October 26 2021).

Atea Pharmaceuticals Inc. (2021). Atea Pharmaceuticals Provides Update and Topline Results for Phase 2 MOONSONG Trial Evaluating AT-527 in the Outpatient Setting. Available at: https://ir.ateapharma.com/news-releases/newsrelease-details/atea-pharmaceuticals-provides-update-and-topline-results-phase2 (Accessed October 26 2021).

Backer, V., Sjöbring, U., Sonne, J., Weiss, A., Hostrup, M., Johansen, H. K., et al. (2021). A Randomized, Double-Blind, Placebo-Controlled Phase 1 Trial of Inhaled and Intranasal Niclosamide: A Broad Spectrum Antiviral Candidate for Treatment of COVID-19. Lancet Reg. Health Eur. 4, 100084. doi:10.1016/j.lanepe.2021.100084 
Baranov, M. V., Bianchi, F., and van den Bogaart, G. (2020). The PIKfyve Inhibitor Apilimod: A Double-Edged Sword against COVID-19. Cells 10 (1), 30. doi:10. 3390/cells 10010030

Blum, V. F., Cimerman, S., Hunter, J. R., Tierno, P., Lacerda, A., Soeiro, A., et al. (2021). Nitazoxanide Superiority to Placebo to Treat Moderate COVID-19 - A Pilot Prove of Concept Randomized Double-Blind Clinical Trial. EClinicalMedicine 37, 100981. doi:10.1016/j.eclinm.2021.100981

Boras, B., Jones, R. M., Anson, B. J., Arenson, D., Aschenbrenner, L., Bakowski, M. A., et al. (2021). Preclinical characterization of an intravenous coronavirus 3CL protease inhibitor for the potential treatment of COVID19. Nat Commun. 12(1), 6055. doi:10.1038/s41467-021-26239-2

Bouhaddou, M., Memon, D., Meyer, B., White, K. M., Rezelj, V. V., Correa Marrero, M., et al. (2020). The Global Phosphorylation Landscape of SARSCoV-2 Infection. Cell 182 (3), 685-712.e19. doi:10.1016/j.cell.2020.06.034

Breining, P., Frølund, A. L., Højen, J. F., Gunst, J. D., Staerke, N. B., Saedder, E., et al. (2021). Camostat Mesylate against SARS-CoV-2 and COVID-19Rationale, Dosing and Safety. Basic Clin. Pharmacol. Toxicol. 128 (2), 204-212. doi: $10.1111 /$ bcpt.13533

Cadegiani, F. A., McCoy, J., Gustavo Wambier, C., Vaño-Galván, S., Shapiro, J., Tosti, A., et al. (2021). Proxalutamide Significantly Accelerates Viral Clearance and Reduces Time to Clinical Remission in Patients with Mild to Moderate COVID-19: Results from a Randomized, DoubleBlinded, Placebo-Controlled Trial. Cureus 13 (2), e13492. doi:10.7759/cureus. 13492

Cai, Q., Yang, M., Liu, D., Chen, J., Shu, D., Xia, J., et al. (2020). Experimental Treatment with Favipiravir for COVID-19: An Open-Label Control Study. Engineering 6 (10), 1192-1198. doi:10.1016/j.eng.2020.03.007

Cai, X., Xu, Y., Cheung, A. K., Tomlinson, R. C., Alcázar-Román, A., Murphy, L., et al. (2013). PIKfyve, a Class III PI Kinase, Is the Target of the Small Molecular IL-12/IL-23 Inhibitor Apilimod and a Player in Toll-like Receptor Signaling. Chem. Biol. 20 (7), 912-921. doi:10.1016/j.chembiol.2013.05.010

Caly, L., Druce, J. D., Catton, M. G., Jans, D. A., and Wagstaff, K. M. (2020). The FDA-Approved Drug Ivermectin Inhibits the Replication of SARS-CoV-2 In Vitro. Antivir. Res 178, 104787. doi:10.1016/j.antiviral.2020.104787

Canard, B., Shannon, A., Fattorini, V., Sama, B., Selisko, B., Feracci, M., et al. (2021). A Dual Mechanism of Action of AT-527 Against SARS-CoV-2 Polymerase.

Cao, B., Wang, Y., Wen, D., Liu, W., Wang, J., Fan, G., et al. (2020). A Trial of Lopinavir-Ritonavir in Adults Hospitalized with Severe Covid-19. N. Engl. J. Med. 382 (19), 1787-1799. doi:10.1056/NEJMoa2001282

Chen, Y., Liu, Q., and Guo, D. (2020). Emerging Coronaviruses: Genome Structure, Replication, and Pathogenesis. J. Med. Virol. 92 (4), 2249-2423. doi:10.1002/ jmv. 26234

Christian, S., Merz, C., Evans, L., Gradl, S., Seidel, H., Friberg, A., et al. (2019). The Novel Dihydroorotate Dehydrogenase (DHODH) Inhibitor BAY 2402234 Triggers Differentiation and Is Effective in the Treatment of Myeloid Malignancies. Leukemia 33 (10), 2403-2415. doi:10.1038/s41375-019-0461-5

Coronavirus Resource Center (2021). Global Map. Available at: https:// coronavirus.jhu.edu/map.html (Accessed November 4, 2021).

Cox, R. M., Wolf, J. D., and Plemper, R. K. (2021). Therapeutically Administered Ribonucleoside Analogue MK-4482/EIDD-2801 Blocks SARS-CoV-2 Transmission in Ferrets. Nat. Microbiol. 6 (1), 11-18. doi:10.1038/s41564020-00835-2

Cvetkovic, R. S., and Goa, K. L. (2003). Lopinavir/ritonavir: a Review of its Use in the Management of HIV Infection. Drugs 63 (8), 769-802. doi:10.2165/ 00003495-200363080-00004

Dai, W., Zhang, B., Jiang, X. M., Su, H., Li, J., Zhao, Y., et al. (2020). Structure-based Design of Antiviral Drug Candidates Targeting the SARS-CoV-2 Main Protease. Science 368 (6497), 1331-1335. doi:10.1126/science.abb4489

Davies, N. G., Abbott, S., Barnard, R. C., Jarvis, C. I., Kucharski, A. J., Munday, J. D., et al. (2021). Estimated Transmissibility and Impact of SARS-CoV-2 Lineage B.1.1.7 in England. Science 372 (6538), eabg3055. doi:10.1126/ science.abg3055

Do, T. N. D., Donckers, K., Vangeel, L., Chatterjee, A. K., Gallay, P. A., Bobardt, M. D., et al. (2021). A Robust SARS-CoV-2 Replication Model in Primary Human Epithelial Cells at the Air Liquid Interface to Assess Antiviral Agents. Antivir. Res 192, 105122. doi:10.1016/j.antiviral.2021.105122
Dömling, A., and Gao, L. (2020). Chemistry and Biology of SARS-CoV-2. Chem 6 (6), 1283-1295. doi:10.1016/j.chempr.2020.04.023

Elfiky, A. A. (2020). Ribavirin, Remdesivir, Sofosbuvir, Galidesivir, and Tenofovir against SARS-CoV-2 RNA Dependent RNA Polymerase (RdRp): A Molecular Docking Study. Life Sci. 253, 117592. doi:10.1016/j. lfs.2020.117592

Faria, N. R., Mellan, T. A., Whittaker, C., Claro, I. M., Candido, D. d. S., Mishra, S., et al. (2021). Genomics and Epidemiology of the P.1 SARS-CoV-2 Lineage in Manaus. Science 372 (6544), 815-821. doi:10.1126/science.abh2644

Food and Drug Administration (2021). Coronavirus Disease 2019 (COVID-19) EUA Information-Drug and Biological Therapeutic Products. Available at: https://www.fda.gov/emergency-preparedness-and-response/mcm-legalregulatory-and-policy-framework/emergency-use-authorization\#coviddrugs (Accessed December 26 2021).

Frieman, M., Yount, B., Heise, M., Kopecky-Bromberg, S. A., Palese, P., and Baric, R. S. (2007). Severe Acute Respiratory Syndrome Coronavirus ORF6 Antagonizes STAT1 Function by Sequestering Nuclear Import Factors on the Rough Endoplasmic reticulum/Golgi Membrane. J. Virol. 81 (18), 9812-9824. doi:10.1128/JVI.01012-07

Fu, D., Cao, R., Zhao, L., Li, W., Zhong, W., and Wen, J. (2020). Oral Favipiravir for Patients with Delayed SARS-CoV-2 Viral RNA Clearance: A Case Series. Crit. Care 24 (1), 578. doi:10.1186/s13054-020-03288-5

Gao, Y., Yan, L., Huang, Y., Liu, F., Zhao, Y., Cao, L., et al. (2020). Structure of the RNA-Dependent RNA Polymerase from COVID-19 Virus. Science 368 (6492), 779-782. doi:10.1126/science.abb7498

Geleris, J., Sun, Y., Platt, J., Zucker, J., Baldwin, M., Hripcsak, G., et al. (2020). Observational Study of Hydroxychloroquine in Hospitalized Patients with Covid-19. N. Engl. J. Med. 382 (25), 2411-2418. doi:10.1056/NEJMoa2012410

Good, S. S., Westover, J., Jung, K. H., Zhou, X. J., Moussa, A., La Colla, P., et al. (2021). AT-527, a Double Prodrug of a Guanosine Nucleotide Analog, Is a Potent Inhibitor of SARS-CoV-2 In Vitro and a Promising Oral Antiviral for Treatment of COVID-19. Antimicrob. Agents Chemother. 65 (4), e02479-02420. doi:10.1128/AAC.02479-20

Good, S. S., Moussa, A., Zhou, X. J., Pietropaolo, K., and Sommadossi, J. P. (2020). Preclinical Evaluation of AT-527, a Novel Guanosine Nucleotide Prodrug with Potent, Pan-Genotypic Activity against Hepatitis C Virus. PLoS One 15(1), e0227104. doi:10.1371/journal.pone.0227104

Gunst, J. D., Staerke, N. B., Pahus, M. H., Kristensen, L. H., Bodilsen, J., Lohse, N., et al. (2021). Efficacy of the TMPRSS2 Inhibitor Camostat Mesilate in Patients Hospitalized with Covid-19-A Double-Blind Randomized Controlled Trial. EClinicalMedicine 35, 100849. doi:10.1016/j.eclinm.2021.100849

Hoffmann, M., Hofmann-Winkler, H., Smith, J. C., Krüger, N., Arora, P., Sørensen, L. K., et al. (2021). Camostat Mesylate Inhibits SARS-CoV-2 Activation by TMPRSS2-Related Proteases and its Metabolite GBPA Exerts Antiviral Activity. EBioMedicine 65, 103255. doi:10.1016/j.ebiom.2021.103255

Hoffmann, M., Kleine-Weber, H., Schroeder, S., Krüger, N., Herrler, T., Erichsen, S., et al. (2020a). SARS-CoV-2 Cell Entry Depends on ACE2 and TMPRSS2 and Is Blocked by a Clinically Proven Protease Inhibitor. Cell 181 (2), 271-e8. doi:10.1016/j.cell.2020.02.052

Hoffmann, M., Mösbauer, K., Hofmann-Winkler, H., Kaul, A., Kleine-Weber, H., Krüger, N., et al. (2020b). Chloroquine Does Not Inhibit Infection of Human Lung Cells with SARS-CoV-2. Nature 585 (7826), 588-590. doi:10.1038/ s41586-020-2575-3

Huang, D., Yu, H., Wang, T., Yang, H., Yao, R., and Liang, Z. (2021). Efficacy and Safety of Umifenovir for Coronavirus Disease 2019 (COVID-19): A Systematic Review and Meta-Analysis. J. Med. Virol. 93 (1), 481-490. doi:10.1002/jmv. 26256

Huang, M., Li, M., Xiao, F., Pang, P., Liang, J., Tang, T., et al. (2020). Preliminary Evidence from a Multicenter Prospective Observational Study of the Safety and Efficacy of Chloroquine for the Treatment of COVID-19. Natl. Sci. Rev. 7 (9), 1428-1436. doi:10.1093/nsr/nwaal13

Inoue, Y., Tanaka, N., Tanaka, Y., Inoue, S., Morita, K., Zhuang, M., et al. (2007). Clathrin-dependent Entry of Severe Acute Respiratory Syndrome Coronavirus into Target Cells Expressing ACE2 with the Cytoplasmic Tail Deleted. J. Virol. 81 (16), 8722-8729. doi:10.1128/jvi.00253-07

Ionescu, M. I. (2020). An Overview of the Crystallized Structures of the SARSCoV-2. Protein J. 39 (6), 600-618. doi:10.1007/s10930-020-09933-w 
Ivashchenko, A. A., Dmitriev, K. A., Vostokova, N. V., Azarova, V. N., Blinow, A. A., Egorova, A. N., et al. (2021). AVIFAVIR for Treatment of Patients with Moderate Coronavirus Disease 2019 (COVID-19): Interim Results of a Phase II/III Multicenter Randomized Clinical Trial. Clin. Infect. Dis. 73 (3), 531-534. doi:10.1093/cid/ciaal176

Jackson, C., Farzan, M., Chen, B., and Choe, H. (2021). Mechanisms of SARS-CoV2 Entry into Cells. Nature Reviews. Mol. Cell Biol. doi:10.1038/s41580-02100418-x

Jeon, S., Ko, M., Lee, J., Choi, I., Byun, S. Y., Park, S., et al. (2020). Identification of Antiviral Drug Candidates against SARS-CoV-2 from FDA-Approved Drugs. Antimicrob. Agents Chemother. 64 (7). doi:10.1128/aac.00819-20

Jin, Z., Du, X., Xu, Y., Deng, Y., Liu, M., Zhao, Y., et al. (2020). Structure of Mpro from SARS-CoV-2 and Discovery of its Inhibitors. Nature 582 (7811), 289-293. doi:10.1038/s41586-020-2223-y

Jockusch, S., Tao, C., Li, X., Anderson, T. K., Chien, M., Kumar, S., et al. (2020a). Triphosphates of the Two Components in DESCOVY and TRUVADA are Inhibitors of the SARS-CoV-2 Polymerase. bioRxiv. doi:10.1101/2020.04.03.022939

Jockusch, S., Tao, C., Li, X., Chien, M., Kumar, S., Morozova, I., et al. (2020b). Sofosbuvir Terminated RNA Is More Resistant to SARS-CoV-2 Proofreader Than RNA Terminated by Remdesivir. Sci. Rep. 10 (1), 16577. doi:10.1038/ s41598-020-73641-9

Kabinger, F., Stiller, C., Schmitzová, J., Dienemann, C., Kokic, G., Hillen, H. S., et al. (2021). Mechanism of Molnupiravir-Induced SARS-CoV-2 Mutagenesis. Nat. Struct. Mol. Biol. 28 (9), 740-746. doi:10.1038/s41594-021-00651-0

Kang, Y. L., Chou, Y. Y., Rothlauf, P. W., Liu, Z., Soh, T. K., Cureton, D., et al. (2020). Inhibition of PIKfyve Kinase Prevents Infection by Zaire Ebolavirus and SARS-CoV-2. Proc. Natl. Acad. Sci. U.S.A. 117 (34), 20803-20813. doi:10.1073/ pnas. 2007837117

Keystone, E. C., Taylor, P. C., Drescher, E., Schlichting, D. E., Beattie, S. D., Berclaz, P. Y., et al. (2015). Safety and Efficacy of Baricitinib at 24 Weeks in Patients with Rheumatoid Arthritis Who Have $\mathrm{Had}$ an Inadequate Response to Methotrexate. Ann. Rheum. Dis. 74 (2), 333-340. doi:10.1136/annrheumdis2014-206478

Kintor Pharma-B (2021). Kintor Pharma Provides Update on One of its Three Multi-Regional Phase 3 Trials of Proxalutamide for COVID-19. Available at: https://en.kintor.com.cn/news/201.html (Accessed December 27 2021).

Kornhuber, J., Hoertel, N., and Gulbins, E. (2021). The Acid Sphingomyelinase/ ceramide System in COVID-19. Mol. Psychiatry. 1-8. Advance online publication. doi:10.1038/s41380-021-01309-5

Lenze, E. J., Mattar, C., Zorumski, C. F., Stevens, A., Schweiger, J., Nicol, G. E., et al. (2020). Fluvoxamine vs Placebo and Clinical Deterioration in Outpatients with Symptomatic COVID-19: A Randomized Clinical Trial. JAMA 324 (22), 2292-2300. doi:10.1001/jama.2020.22760

Li, W., Moore, M. J., Vasilieva, N., Sui, J., Wong, S. K., Berne, M. A., et al. (2003). Angiotensin-converting Enzyme 2 Is a Functional Receptor for the SARS Coronavirus. Nature 426 (6965), 450-454. doi:10.1038/nature02145

Liu, J., Cao, R., Xu, M., Wang, X., Zhang, H., Hu, H., et al. (2020). Hydroxychloroquine, a Less Toxic Derivative of Chloroquine, is Effective in Inhibiting SARS-CoV-2 Infection In Vitro. Cell Discov 6, 16. doi:10.1038/ s41421-020-0156-0

Lokhande, A. S., and Devarajan, P. V. (2021). A Review on Possible Mechanistic Insights of Nitazoxanide for Repurposing in COVID-19. Eur. J. Pharmacol. 891, 173748. doi:10.1016/j.ejphar.2020.173748

Lu, R., Zhao, X., Li, J., Niu, P., Yang, B., Wu, H., et al. (2020). Genomic Characterisation and Epidemiology of 2019 Novel Coronavirus: Implications for Virus Origins and Receptor Binding. Lancet 395 (10224), 565-574. doi:10. 1016/s0140-6736(20)30251-8

Luban, J., Sattler, R., Mühlberger, E., Graci, J. D., Cao, L., Weetall, M., et al. (2021). The DHODH Inhibitor PTC299 Arrests SARS-CoV-2 Replication and Suppresses Induction of Inflammatory Cytokines. Virus. Res. 292, 198246. doi:10.1016/j.virusres.2020.198246

Mahase, E. (2021). Covid-19: Pfizer's Paxlovid Is 89\% Effective in Patients at Risk of Serious Illness, Company Reports. BMJ 375, n2713. doi:10.1136/bmj. n2713

Mahmoud, D. B., Shitu, Z., and Mostafa, A. (2020). Drug Repurposing of Nitazoxanide: Can it Be an Effective Therapy for COVID-19. J. Genet. Eng. Biotechnol. 18 (1), 35. doi:10.1186/s43141-020-00055-5
Marconi, V. C., Ramanan, A. V., de Bono, S., Kartman, C. E., Krishnan, V., Liao, R., et al. (2021). Efficacy and Safety of Baricitinib for the Treatment of Hospitalised Adults with COVID-19 (COV-BARRIER): a Randomised, Double-Blind, Parallel-Group, Placebo-Controlled Phase 3 Trial. Lancet Respir. Med. 9 (12), 1407-1418. doi:10.1016/S2213-2600(21)00331-3

Merck (2021a). Merck and Ridgeback Statement on Positive FDA Advisory Committee Vote for Investigational Oral Antiviral Molnupiravir for Treatment of Mild to Moderate COVID-19 in High Risk Adults. Available at: https://www. merck.com/news/merck-and-ridgeback-statement-on-positive-fda-advisorycommittee-vote-for-investigational-oral-antiviral-molnupiravir-for-treatment-ofmild-to-moderate-covid-19-in-high-risk-adults/ (Accessed November 30 2021).

Merck (2021b). Merck and Ridgeback's Molnupiravir, an Oral COVID-19 Antiviral Medicine, Receives First Authorization in the World. Available at: https://www.merck.com/news/merck-and-ridgebacks-molnupiravir-anoral-covid-19-antiviral-medicine-receives-first-authorization-in-the-world/ (Accessed October 26 2021).

Mesci, P., Macia, A., Saleh, A., Martin-Sancho, L., Yin, X., Snethlage, C., et al. (2020). Sofosbuvir Protects Human Brain Organoids against SARS-CoV-2. bioRxiv. doi:10.1101/2020.05.30.125856

Mollica, V., Rizzo, A., and Massari, F. (2020). The Pivotal Role of TMPRSS2 in Coronavirus Disease 2019 and Prostate Cancer. Future Oncol. 16 (27), 2029-2033. doi:10.2217/fon-2020-0571

Muramatsu, T., Takemoto, C., Kim, Y. T., Wang, H., Nishii, W., Terada, T., et al. (2016). SARS-CoV 3CL Protease Cleaves its C-Terminal Autoprocessing Site by Novel Subsite Cooperativity. Proc. Natl. Acad. Sci. U S A. 113 (46), 12997-13002. doi:10.1073/pnas.1601327113

National Institutes of Health (2020). NIH Halts Clinical Trial of Hydroxychloroquine. Available at: https://www.nih.gov/news-events/news-releases/nih-halts-clinicaltrial-hydroxychloroquine (Accessed October 26 2021).

Naydenova, K., Muir, K. W., Wu, L. F., Zhang, Z., Coscia, F., Peet, M. J., et al. (2021). Structure of the SARS-CoV-2 RNA-dependent RNA Polymerase in the Presence of Favipiravir-RTP. Proc. Natl. Acad. Sci. U S A. 118 (7), 946118. doi:10.1073/pnas.2021946118

Pan, H., Pan, H., Peto, R., Henao-Restrepo, A. M., Preziosi, M. P., Sathiyamoorthy, V., et al. (2021). Repurposed Antiviral Drugs for Covid-19 - Interim WHO Solidarity Trial Results. N. Engl. J. Med. 384 (6), 497-511. doi:10.1056/ NEJMoa2023184

Peng, Q., Peng, R., Yuan, B., Wang, M., Zhao, J., Fu, L., et al. (2021). Structural Basis of SARS-CoV-2 Polymerase Inhibition by Favipiravir. Innovation 2 (1), 100080. doi:10.1016/j.xinn.2021.100080

Persoons, L., Vanderlinden, E., Vangeel, L., Wang, X., Do, N. D. T., Foo, S. C., et al. (2021). Broad Spectrum Anti-coronavirus Activity of a Series of Anti-malaria Quinoline Analogues. Antivir. Res. 193, 105127. doi:10.1016/j.antiviral.2021. 105127

Pfizer Inc. (2021). PFIZER'S Novel Covid-19 Oral Antiviral Treatment Candidate Reduced Risk of Hospitalization or Death By 89\% In Interim Analysis of Phase 2/3 Epic-Hr Study. Available at: https://www.pfizer.com/news/press-release/ press-release-detail/pfizers-novel-covid-19-oral-antiviral-treatment-candidate (Accessed November 05 2021).

Plaze, M., Attali, D., Petit, A. C., Blatzer, M., Simon-Loriere, E., Vinckier, F., et al. (2020). Repurposing Chlorpromazine to Treat COVID-19: The reCoVery Study. Encephale 46 (3), 169-172. doi:10.1016/j.encep.2020. 05.006

Plaze, M., Attali, D., Prot, M., Petit, A. C., Blatzer, M., Vinckier, F., et al. (2021). Inhibition of the Replication of SARS-CoV-2 in Human Cells by the FDAApproved Drug Chlorpromazine. Int. J. Antimicrob. Agents 57 (3), 106274. doi:10.1016/j.ijantimicag.2020.106274

Prabhakara, C., Godbole, R., Sil, P., Jahnavi, S., Gulzar, S. E., van Zanten, T. S., et al. (2021). Strategies to target SARS-CoV-2 entry and infection using dual mechanisms of inhibition by acidification inhibitors. PLoS Pathog 17 (7), e1009706. doi:10.1371/journal.ppat.1009706

PRINCIPLE (2021). Favipiravir to Be Investigated as a Possible COVID-19 Treatment for at-home Recovery in the PRINCIPLE Trial. Available at: https://www.principletrial.org/news/favipiravir-to-be-investigated-as-a-possiblecovid-19-treatment-for-at-home-recovery-in-theprinciple-trial (Accessed October 26, 2021).

Rafiee, L., Hajhashemi, V., and Javanmard, S. H. (2016). Fluvoxamine Inhibits Some Inflammatory Genes Expression in LPS/stimulated Human Endothelial 
Cells, U937 Macrophages, and Carrageenan-Induced Paw Edema in Rat. Iran J. Basic Med. Sci. 19 (9), 977-984.

Ramos-Guzmán, C. A., Ruiz-Pernía, J. J., and Tuñón, I. (2021). Computational Simulations on the Binding and Reactivity of a Nitrile Inhibitor of the SARSCoV-2 Main Protease. Chem. Commun. 57 (72), 9096-9099. doi:10.1039/ d1cc03953a

Reis, G., Dos Santos Moreira-Silva, E. A., Silva, D. C. M., Thabane, L., Milagres, A. C., Ferreira, T. S., et al. (2022). Effect of Early Treatment with Fluvoxamine on Risk of Emergency Care and Hospitalisation Among Patients with COVID-19: the TOGETHER Randomised, Platform Clinical Trial. Lancet Glob. Health 10 (1), e42-e51. doi:10.1016/S2214109X(21)00448-4

Ren, Z., Luo, H., Yu, Z., Song, J., Liang, L., Wang, L., et al. (2020). A Randomized, Open-Label, Controlled Clinical Trial of Azvudine Tablets in the Treatment of Mild and Common COVID-19, A Pilot Study. Adv. Sci. 7 (19), 2001435. doi:10. 1002/advs.202001435

Rolain, J. M., Colson, P., and Raoult, D. (2007). Recycling of Chloroquine and its Hydroxyl Analogue to Face Bacterial, Fungal and Viral Infections in the 21st century. Int. J. Antimicrob. Agents 30 (4), 297-308. doi:10.1016/j.ijantimicag. 2007.05.015

Roozbeh, F., Saeedi, M., Alizadeh-Navaei, R., Hedayatizadeh-Omran, A., Merat, S., Wentzel, H., et al. (2021). Sofosbuvir and Daclatasvir for the Treatment of COVID-19 Outpatients: a Double-Blind, Randomized Controlled Trial. J. Antimicrob. Chemother. 76 (3), 753-757. doi:10.1093/jac/dkaa501

Rosen, D. A., Seki, S. M., Fernández-Castañeda, A., Beiter, R. M., Eccles, J. D., Woodfolk, J. A., et al. (2019). Modulation of the Sigma-1 Receptor-IRE1 Pathway Is Beneficial in Preclinical Models of Inflammation and Sepsis. Sci. Transl Med.11, 478. doi:10.1126/scitranslmed.aau5266

Sacramento, C. Q., Fintelman-Rodrigues, N., Temerozo, J. R., Da Silva, A., Dias, S., da Silva, C., et al. (2021). In vitro antiviral activity of the anti-HCV drugs daclatasvir and sofosbuvir against SARS-CoV-2, the aetiological agent of COVID-19. J Antimicrob Chemother. 76 (7), 1874-1885. doi:10.1093/jacl dkab072

Sadeghi, A., Ali Asgari, A., Norouzi, A., Kheiri, Z., Anushirvani, A., Montazeri, M., et al. (2020). Sofosbuvir and Daclatasvir Compared with Standard of Care in the Treatment of Patients Admitted to Hospital with Moderate or Severe Coronavirus Infection (COVID-19): A Randomized Controlled Trial. J. Antimicrob. Chemother. 75 (11), 3379-3385. doi:10.1093/jac/dkaa334

Shahab, S., and Sheikhi, M. (2020). Triazavirin - Potential Inhibitor for 2019-nCoV Coronavirus M Protease: A DFT Study. Curr. Mol. Med. 21 (8), 645-654. doi:10. 2174/1566524020666200521075848

Shannon, A., Selisko, B., Le, N. T., Huchting, J., Touret, F., Piorkowski, G., et al. (2020). Rapid Incorporation of Favipiravir by the Fast and Permissive Viral RNA Polymerase Complex Results in SARS-CoV-2 Lethal Mutagenesis. Nat. Commun. 11 (1), 4682. doi:10.1038/s41467-020-18463-Z

Sheahan, T. P., Sims, A. C., Zhou, S., Graham, R. L., Pruijssers, A. J., Agostini, M. L., et al. (2020). An Orally Bioavailable Broad-Spectrum Antiviral Inhibits SARSCoV-2 in Human Airway Epithelial Cell Cultures and Multiple Coronaviruses in Mice. Sci. Transl. Med. 12(541), eabb5883. doi:10.1126/scitranslmed.abb5883

Shinkai, M., Tsushima, K., Tanaka, S., Hagiwara, E., Tarumoto, N., Kawada, I., et al. (2021). Efficacy and Safety of Favipiravir in Moderate COVID-19 Pneumonia Patients without Oxygen Therapy: A Randomized, Phase III Clinical Trial. Infect. Dis. Ther. 10 (4), 2489-2509. doi:10.1007/s40121-021-00517-4

Shionogi \& Co., Ltd. (2021a). Notice Regarding the Initiation of a Phase 1 Clinical Trial for a COVID-19 Therapeutic Agent in Japan. Available at: https://www. shionogi.com/global/en/news/2021/07/e-210726.html (Accessed October 26, 2021).

Shionogi \& Co., Ltd. (2021b). Notice Regarding the Initiation of a Phase 2/3 Clinical Trial for a COVID-19 Therapeutic Agent in Japan. Available at: https:// www.shionogi.com/global/en/news/2021/09/e-210928.html (Accessed October 26 2021).

Shionogi \& Co., Ltd. (2021c). Shionogi Presents COVID-19 Therapeutic Agent Results at the ISIRV-WHO Virtual Conference. Available at: https://www. shionogi.com/global/en/news/2021/10/e-211021_2.html (Accessed November 05 2021).

Song, Z. (2020). Application of 2, 4, 5-trisubstituted 1, 2, 4-trizolone in Preparation of Antiviral Drug.
Sun, W., Zhang, X., Cummings, M. D., Albarazanji, K., Wu, J., Wang, M., et al. (2020). Targeting Enteropeptidase with Reversible Covalent Inhibitors to Achieve Metabolic Benefits. J. Pharmacol. Exp. Ther. 375 (3), 510-521. doi:10.1124/jpet.120.000219

Talevi, A., and Bellera, C. L. (2020). Challenges and Opportunities with Drug Repurposing: Finding Strategies to Find Alternative Uses of Therapeutics. Expert Opin. Drug Discov. 15 (4), 397-401. doi:10.1080/17460441.2020. 1704729

Taylor, R., Kotian, P., Warren, T., Panchal, R., Bavari, S., Julander, J., et al. (2016). BCX4430 - A Broad-Spectrum Antiviral Adenosine Nucleoside Analog under Development for the Treatment of Ebola Virus Disease. J. Infect. Public Health 9 (3), 220-226. doi:10.1016/j.jiph.2016.04.002

Toots, M., Yoon, J. J., Hart, M., Natchus, M. G., Painter, G. R., and Plemper, R. K. (2020). Quantitative Efficacy Paradigms of the Influenza Clinical Drug Candidate EIDD-2801 in the Ferret Model. Transl Res. 218, 16-28. doi:10. 1016/j.trsl.2019.12.002

Uzunova, K., Filipova, E., Pavlova, V., and Vekov, T. (2020). Insights into Antiviral Mechanisms of Remdesivir, Lopinavir/ritonavir and Chloroquine/ hydroxychloroquine Affecting the New SARS-CoV-2. Biomed. Pharmacother. 131, 110668. doi:10.1016/j.biopha.2020.110668

Vankadari, N. (2020). Arbidol: A Potential Antiviral Drug for the Treatment of SARS-CoV-2 by Blocking Trimerization of the Spike Glycoprotein. Int. J. Antimicrob. Agents 56 (2), 105998. doi:10.1016/j.ijantimicag.2020.105998

Vincent, M. J., Bergeron, E., Benjannet, S., Erickson, B. R., Rollin, P. E., Ksiazek, T. G., et al. (2005). Chloroquine Is a Potent Inhibitor of SARS Coronavirus Infection and Spread. Virol. J. 2, 69. doi:10.1186/1743-422x-2-69

Wahl, A., Gralinski, L. E., Johnson, C. E., Yao, W., Kovarova, M., Dinnon, K. H., 3 rd, et al. (2021). SARS-CoV-2 Infection Is Effectively Treated and Prevented by EIDD-2801. Nature 591 (7850), 451-457. doi:10.1038/s41586-02103312-w

Wang, C., Horby, P. W., Hayden, F. G., and Gao, G. F. (2020). A Novel Coronavirus Outbreak of Global Health Concern. Lancet 395 (10223), 470-473. doi:10.1016/ s0140-6736(20)30185-9

Wang, K., Chen, W., Zhang, Z., Deng, Y., Lian, J. Q., Du, P., et al. (2020). CD147-spike Protein is a Novel Route for SARS-CoV-2 Infection to Host Cells. Signal. Transduct Target. Ther. 5 (1), 283. doi:10.1038/s41392-02000426-x

Wang, M., Cao, R., Zhang, L., Yang, X., Liu, J., Xu, M., et al. (2020). Remdesivir and Chloroquine Effectively Inhibit the Recently Emerged Novel Coronavirus (2019$\mathrm{nCoV}$ ) In Vitro. Cell Res 30 (3), 269-271. doi:10.1038/s41422-020-0282-0

Wang, X., Cao, R., Zhang, H., Liu, J., Xu, M., Hu, H., et al. (2020). The Antiinfluenza Virus Drug, Arbidol Is an Efficient Inhibitor of SARS-CoV-2 In Vitro. Cell Discov. 6, 28. doi:10.1038/s41421-020-0169-8

White, K. M., Rosales, R., Yildiz, S., Kehrer, T., Miorin, L., Moreno, E., et al. (2021). Plitidepsin Has Potent Preclinical Efficacy Against SARS-CoV-2 by Targeting the Host Protein eEF1A. Science 371 (6532), 926-931. doi:10.1126/science. abf 4058

Wong, J. P., and Damania, B. (2021). SARS-CoV-2 Dependence on Host Pathways. Science 371 (6532), 884-885. doi:10.1126/science.abg6837

World-Health-Organization (2021). COVID-19 Weekly Epidemiological Update. Available at: https://www.who.int/publications/m/item/weekly-epidemiologicalupdate-on-covid-19--31-august-2021 (Accessed October 26, 2021).

Wu, C. Y., Jan, J. T., Ma, S. H., Kuo, C. J., Juan, H. F., Cheng, Y. S., et al. (2004). Small Molecules Targeting Severe Acute Respiratory Syndrome Human Coronavirus. Proc. Natl. Acad. Sci. U.S.A. 101 (27), 10012-10017. doi:10. 1073/pnas.0403596101

Wu, F., Zhao, S., Yu, B., Chen, Y. M., Wang, W., Song, Z. G., et al. (2020). A New Coronavirus Associated with Human Respiratory Disease in China. Nature 579 (7798), 265-269. doi:10.1038/s41586-020-2008-3

Wu, S., Miao, L., Zhou, Q., Gao, C., Liu, J., Zhan, Q., et al. (2020). Suppression of Androgen Receptor (AR)-ACE2/TMPRSS2 Axis by AR Antagonists May Be Therapeutically Beneficial for Male COVID-19 Patients. Available at: https:// ssrn.com/abstract=3580526 (Accessed April 20, 2020).

Wu, X., Yu, K., Wang, Y., Xu, W., Ma, H., Hou, Y., et al. (2020). Efficacy and Safety of Triazavirin Therapy for Coronavirus Disease 2019: A Pilot Randomized Controlled Trial. Engineering 6 (10), 1185-1191. doi:10. 1016/j.eng.2020.08.011 
Xie, Y., Yin, W., Zhang, Y., Shang, W., Wang, Z., Luan, X., et al. (2021). Design and Development of an Oral Remdesivir Derivative VV116 against SARS-CoV-2. Cell Res. 31 (11), 1212-1214. doi:10.1038/s41422-021-00570-1

Xiong, R., Zhang, L., Li, S., Sun, Y., Ding, M., Wang, Y., et al. (2020). Novel and Potent Inhibitors Targeting DHODH Are Broad-Spectrum Antivirals against RNA Viruses Including Newly-Emerged Coronavirus SARS-CoV-2. Protein Cell 11 (10), 723-739. doi:10.1007/s13238-020-00768-w

Xu, X., Chen, P., Wang, J., Feng, J., Zhou, H., Li, X., et al. (2020). Evolution of the Novel Coronavirus from the Ongoing Wuhan Outbreak and Modeling of its Spike Protein for Risk of Human Transmission. Sci. China Life Sci. 63 (3), 457-460. doi:10.1007/s11427-020-1637-5

Yang, S. N. Y., Atkinson, S. C., Wang, C., Lee, A., Bogoyevitch, M. A., Borg, N. A., et al. (2020). The Broad Spectrum Antiviral Ivermectin Targets the Host Nuclear Transport Importin $\alpha / \beta 1$ Heterodimer. Antivir. Res 177, 104760. doi:10.1016/j.antiviral.2020.104760

Yavuz, S., and Komsuoğlu Çelikyurt, F. I. (2021). Antiviral Treatment of COVID-19: An Update. Turk J. Med. Sci. 51(SI), 3372-3390. doi:10.3906/ sag-2106-250

Yin, W., Mao, C., Luan, X., Shen, D. D., Shen, Q., Su, H., et al. (2020). Structural Basis for Inhibition of the RNA-dependent RNA Polymerase from SARS-CoV2 by Remdesivir. Science 368 (6498), 1499-1504. doi:10.1126/science.abc1560

Yu, B., and Chang, J. (2020). Azvudine (FNC): A Promising Clinical Candidate for COVID-19 Treatment. Signal. Transduct. Target. Ther. 5 (1), 236. doi:10.1038/ s41392-020-00351-z

Zhang, C., Jin, H., Wen, Y. F., and Yin, G. (2021). Efficacy of COVID-19 Treatments: A Bayesian Network Meta-Analysis of Randomized Controlled Trials. Front. Public Health 9, 729559. doi:10.3389/fpubh. 2021.729559

Zhang, W. F., Stephen, P., Thériault, J. F., Wang, R., and Lin, S. X. (2020). Novel Coronavirus Polymerase and Nucleotidyl-Transferase Structures: Potential to Target New Outbreaks. J. Phys. Chem. Lett. 11 (11), 4430-4435. doi:10.1021/acs. jpclett.0c00571

Zhang, X., Zhang, Y., Qiao, W., Zhang, J., and Qi, Z. (2020). Baricitinib, a Drug with Potential Effect to Prevent SARS-COV-2 from Entering Target Cells and Control Cytokine Storm Induced by COVID-19. Int. Immunopharmacol. 86, 106749. doi:10.1016/j.intimp.2020.106749
Zhao, L., and Zhong, W. (2021). Mechanism of Action of Favipiravir against SARSCoV-2: Mutagenesis or Chain Termination. Innovation 2 (4). doi:10.1016/j. xinn.2021.100165

Zhou, P., Yang, X. L., Wang, X. G., Hu, B., Zhang, L., Zhang, W., et al. (2020). A Pneumonia Outbreak Associated with a New Coronavirus of Probable Bat Origin. Nature 579 (7798), 270-273. doi:10.1038/s41586-020-2012-7

Zhou, S., Hill, C. S., Sarkar, S., Tse, L. V., Woodburn, B. M. D., Schinazi, R. F., et al. (2021). $\quad \beta$-d-N4-hydroxycytidine Inhibits SARS-CoV-2 through Lethal Mutagenesis but Is Also Mutagenic to Mammalian Cells. J. Infect. Dis. 224 (3), 415-419. doi:10.1093/infdis/jiab247

Zhu, G., Zhu, C., Zhu, Y., and Sun, F. (2020). Minireview of Progress in the Structural Study of SARS-CoV-2 Proteins. Curr. Res. Microb. Sci. 1, 53-61. doi:10.1016/j.crmicr.2020.06.003

Zhu, N., Zhang, D., Wang, W., Li, X., Yang, B., Song, J., et al. (2020). A Novel Coronavirus from Patients with Pneumonia in China, 2019. N. Engl. J. Med. 382 (8), 727-733. doi:10.1056/NEJMoa2001017

Conflict of Interest: Author LZ was employed by the company Beijing Sunho Pharmaceutical Co., Ltd.

The remaining authors declare that the research was conducted in the absence of any commercial or financial relationships that could be construed as a potential conflict of interest.

Publisher's Note: All claims expressed in this article are solely those of the authors and do not necessarily represent those of their affiliated organizations, or those of the publisher, the editors and the reviewers. Any product that may be evaluated in this article, or claim that may be made by its manufacturer, is not guaranteed or endorsed by the publisher.

Copyright (c) 2022 Zhao, Li and Zhong. This is an open-access article distributed under the terms of the Creative Commons Attribution License (CC BY). The use, distribution or reproduction in other forums is permitted, provided the original author(s) and the copyright owner(s) are credited and that the original publication in this journal is cited, in accordance with accepted academic practice. No use, distribution or reproduction is permitted which does not comply with these terms. 